\title{
Fast computation of optimized electrode arrays for 2D resistivity surveys
}

\author{
M.H.Loke ${ }^{1}$, P. Wilkinson ${ }^{2}$, J. Chambers ${ }^{2}$ \\ ${ }^{1}$ Geotomo Software, 115 Cangkat Minden Jalan 5, Minden Heights, 11700 Gelugor, Penang, Malaysia. \\ email : drmhloke@yahoo.com, Tel : +60 4 6574525, Fax : +60 4 6588437. Corresponding author. \\ 2 British Geological Survey, Kingsley Dunham Centre, Keyworth, Nottingham, United Kingdom \\ NG12 5GG. email : pbw@bgs.ac.uk, jecha@bgs.ac.uk
}

\begin{abstract}
Four different methods to automatically select an optimal set of array configurations that gives the maximum resolution of the subsurface with a limited number of measurements for $2 \mathrm{D}$ electrical imaging surveys were tested. The first method (CR method) directly calculates the change in the model resolution for each new array added to the base data set, and uses this to select array configurations that gave the maximum model resolution. However this method is the slowest. The computer code for calculating the rank one updates used by the CR method was optimized to reduce the computer time by about eighty times. The sequence of calculations needed was modified to reduce the traffic between the computer main memory and the CPU registers. Further code optimizations were made to take advantage of the parallel processing capabilities of modern CPUs. The second (ETH) and third (BGS) methods use approximations based on the sensitivity values to estimate the change in the model resolution matrix. The ETH and BGS methods respectively use the first and second power of the sensitivity values to calculate an approximation of the model resolution. Both methods are about an order of magnitude faster than the CR method. The results obtained by the BGS method are significantly better than the ETH method, and it approaches that of the CR method. The fourth method (BGS-CR) uses a combination of the BGS and CR methods. It produces results that are almost identical to the CR method but it is several times faster. The different methods were tested using data from synthetic models and field surveys. The
\end{abstract}


models obtained from the inversion of the data sets generated by the four different methods confirm that the models generated by the CR method have the best resolution, followed by the BGS-CR, BGS and ETH methods.

Keywords : resistivity; 2D; array; optimization; model; resolution 


\section{INTRODUCTION}

Over the last decade the resistivity method has undergone a renaissance with the development of multielectrode resistivity-meter systems and efficient 2D and 3D inversion schemes. Electrical imaging (or tomography) surveys are now widely used in diverse engineering and environmental problems, hydrological, agricultural, mineral and even hydrocarbon exploration (Dahlin, 1996; Seaton and Burbey, 2000; Bauman, 2005). Most surveys have been carried out using the traditional arrays such as the Wenner, Schlumberger, pole-pole, dipole-dipole and pole-dipole arrays. The general characteristics in terms of resolution, depth of investigation and noise sensitivity of these arrays are relatively well known (Barker, 1979, 1989; Dahlin and Zhou, 2004). In general, the Wenner and Schlumberger arrays are less sensitive to noise and have better resolution for horizontal structures. The dipole-dipole and pole-dipole arrays have lower signal-to-noise ratios and have better resolution for vertical structures. However, in many survey areas, good resolution in both directions is required. Modern computer-controlled multielectrode systems can measure thousands of readings. The survey time, and thus the expense, is affected by the number of measurements required to meet the survey objective. Ideally, the array configurations used should maximize the resolution of the inversion model obtained from the field survey using a limited number of measurements. An important development was made by Stummer et al. (2004) who proposed a fast and accurate method to automatically select the arrays that would maximize the model resolution. This approach used the sensitivity distribution to provide an approximation to the model resolution matrix. This was further improved by Wilkinson et al. (2006a) who proposed two new methods to improve the quality of the optimized array configurations. One method directly recalculates the model resolution (the 'Compare R' method) for each new array and produces array sets with the maximum model resolution. However, the computer time required by this method was about two orders of magnitude longer that the other methods (about 6 hours on a $3 \mathrm{Ghz}$ PC for a survey line with 30 electrodes). The second method (the 'Modified GF' method) used a modified estimate of the change in 
the model resolution for a new array. It was able to generate arrays sets that have higher model resolution than the original method by Stummer et al. (2004) while taking less computer time (about 3.9 minutes on the same computer).

As the 'Compare R" proved to be the optimal technique, and the standard to which other methods are compared, efforts were made in this research to reduce the computer time required so that it can be used for larger problems. A modification to the method proposed by Stummer et al. (2004) was also made to reduce the computer time it requires. Finally, a combination of the 'Modified GF' and 'Compare R' methods to combine the best features of both techniques was investigated.

The following section gives a brief review of the linearized least-squares inversion method and its relationship to the model resolution matrix. This is followed by a description of the four different methods to automatically generate the optimized arrays, and a comparison of the results. Finally, we test the optimized arrays with synthetic and field data sets.

\section{THEORY}

In the 2D resistivity method, a large number of measurements are made using different electrode configurations in order to map the subsurface resistivity at different depths below the survey line. We then attempt to determine the true resistivity of the subsurface from the apparent resistivity measurements. One method that is widely used is the linearized least-squares optimization method (deGroot-Hedlin and Constable, 1990; Ellis and Oldenburg, 1994) where the relationship between the measured data and model parameters is given by the following equation.

$$
\left(\mathbf{G}^{\mathrm{T}} \mathbf{G}+\mathbf{C}\right) \Delta \mathbf{r}_{\mathrm{i}}=\mathbf{G}^{\mathrm{T}} \mathbf{g}-\mathbf{C} \mathbf{r}_{\mathrm{i}-1},
$$

The Jacobian matrix $\mathbf{G}$ contains the logarithmic sensitivities of the measurements with respect to the model parameters. The matrix $\mathbf{C}$ contains the damping factors, roughness filters and other constraints, while $\mathbf{g}$ is the data misfit vector containing the difference between the logarithms of the measured and 
calculated apparent resistivity values. $\mathbf{r}_{\mathbf{i}-1}$ is the model parameter vector (the logarithm of the model resistivity values) for the previous iteration, while is $\Delta \mathbf{r}_{\mathbf{i}}$ is change in the model parameters. Using linear approximations, it can be shown an estimate of the model resolution matrix (Menke, 1984) is given by

$$
\mathbf{R}=\left(\mathbf{G}^{\mathrm{T}} \mathbf{G}+\mathbf{C}\right)^{-1} \mathbf{G}^{\mathrm{T}} \mathbf{G}
$$

The model resolution matrix may be viewed as a filter through which the inversion method attempts to resolve the subsurface resistivity (Day-Lewis et al., 2005). In the ideal case with perfect resolution, the elements of the main diagonal $\left(R_{j j}\right)$ are 1 while the off-diagonal elements are 0 . In practical cases, the leading diagonal elements are between 0 and 1 . The array optimization methods attempt to select the array configurations that maximize the values of the leading diagonal elements of the resolution matrix. We use the approach by Wilkinson et al. (2006a) in calculating the sensitivity values of a homogeneous model for the Jacobian matrix $\mathbf{G}$, and a simple Marquardt-Levenberg constraint for the $\mathbf{C}$ matrix (i.e. $\mathbf{C}=\lambda \mathbf{I}$ where $\lambda$ is a damping factor and $\mathbf{I}$ is the identity matrix).

For a system with $\mathrm{N}$ electrodes, there are $N(N-1)(N-2)(N-3) / 8$ independent four-electrode configurations (Xu and Noel, 1991). This gives a total of 82215 possible configurations for a system with 30 electrodes. To reduce the number of possible arrays, Stummer et al. (2004) excluded configurations that are likely to reduce the stability of the inversion. These include configurations of the Gamma type with crossed current and potential electrodes (Figure 1), and configurations with large geometric factors.

Carpenter and Habberjam (1956) showed that there are three possible arrangements for an electrode configuration with four electrodes (Figure 1). The three arrangements are the Alpha (with the potential electrodes nested between the current electrodes), Beta (with separate current and potential dipoles) and the Gamma (with interleaved current and potential electrodes). They also showed that the resistance value measured by the Gamma configuration is equals to the difference of resistances measured with the Alpha and Beta configurations. Thus, in theory, the Gamma configuration is not an 
independent arrangement as it can be derived from the Alpha and Beta measurements. This further justifies the decision by Stummer et al. (2004) and Wilkinson et al. (2006a) in excluding the Gamma configurations from the arrays considered in their optimization schemes.

Figure 1 here.

All configurations with geometric factors larger than that for a dipole-dipole array where the maximum dipole separation is greater than six times the current/potential dipole length (i.e. with an ' $a$ ' spacing of 1 and ' $n$ ' level of 6) are also excluded. In some field surveys, particularly those using large electrode spacings, it might be necessary reduce the maximum ' $n$ ' value to reduce the random noise in the measurements. However, for the following tests, a maximum value of 6 was used for the ' $n$ ' factor. The choice of this maximum geometric factor was to be consistent with earlier tests by Stummer et al. (2004) and Wilkinson et al. (2006a) so that the results from this paper can be directly compared. However, a larger value for the maximum geometric factor can be used in situations with sufficiently low noise levels so as to include more possible array configurations. This was done in generating the arrays configurations in the field test that is described in a later part of this paper.

The configurations left after excluding the less stable configurations are called the 'comprehensive' data set. The 'comprehensive' data set for a survey line with 30 electrodes has 51283 unique configurations (compared to 82215 in the original set). The optimization task is to select a subset of the comprehensive set that will maximize the model resolution subject to practical constraints (such as the maximum number of measurements that can be made within the available survey time). We follow the approach by Wilkinson et al. (2006a) and Stummer et al. (2004) in using a local optimization procedure. Initially, a small starting base data set is selected. This normally consists of the dipole-dipole configurations with an ' $a$ ' spacing of 1 and ' $n$ ' levels of 1 to 6 . The change in the model resolution matrix $\mathbf{R}$ (or some estimate of the change) for each new configuration added to the base data set is then calculated. The configurations that result in the largest increase in the model resolution, and have a 
suitable degree of orthogonality to the existing configurations, are then added to the base data set. Three different methods to calculate or estimate the change in the model resolution were reviewed by Wilkinson et al. (2006a).

\section{METHODS OF ARRAYS SELECTION}

\section{Method 1 - Compare R (CR)}

This method directly recalculates the model resolution matrix when a single array configuration is added to the existing base data set. The Sherman-Morrison Rank-1 update is used to calculate the main diagonal elements of the model resolution matrix of the base set plus the test configuration (Wilkinson et al., 2006a). To simplify the following discussion, we rewrite equation (2) in the following form.

$$
\mathbf{R}_{\mathbf{b}}=\mathbf{B}_{\mathbf{b}} \mathbf{A}_{\mathbf{b}}
$$

where $\mathbf{R}_{\mathbf{b}}$ is the model resolution matrix of the base data set, $\mathbf{A}=\mathbf{G}^{\mathrm{T}} \mathbf{G}$ and $\mathbf{B}=\left(\mathbf{G}^{\mathrm{T}} \mathbf{G}+\mathbf{C}\right)^{-1}$. The following set of updating formulae (Press et al., 1992) is used to calculate the change in $\mathbf{R}$ when a new array configuration is added to the base data set.

$$
\begin{aligned}
& \mathbf{A}_{\mathbf{b}+1}=\mathbf{A}_{\mathbf{b}}+\mathbf{g ~ g}^{\mathbf{T}} \\
& \mathbf{B}_{\mathbf{b}+1}=\mathbf{B}_{\mathbf{b}}-\frac{\mathbf{z z}^{\mathbf{T}}}{1+\mu} \\
& \mathbf{R}_{\mathbf{b}+\mathbf{1}}=\mathbf{B}_{\mathbf{b}+\mathbf{1}} \mathbf{A}_{\mathbf{b}+\mathbf{1}}
\end{aligned}
$$

$\mathbf{g}$ is the vector containing logarithmic sensitivity values of the test configuration, $\mathbf{z}=\mathbf{B}_{\mathbf{b}} \mathbf{g}$ and $\mu=\mathbf{g . z}$. $\mathbf{R}_{\mathbf{b + 1}}$ is the resolution of the base data set plus the test configuration (Wilkinson et al., 2006a). The following function, $F^{C R}$, is used the rank the improvement in the model resolution due to the addition of the test configuration. It is given by 


$$
F^{C R}=\frac{1}{m} \sum_{j=1}^{m} \frac{R_{b+1}(j, j)}{R_{b}(j, j)}
$$

where $m$ is the number of model cells, and only the main diagonal elements of the $\mathbf{R}$ matrix are used. This method was found to give the best results (in terms of improvement in the model resolution) but was about two orders of magnitude slower than the other methods. Wilkinson et al. (2006a) reported a calculation time of 6 hours for a survey system with 30 electrodes on a $3 \mathrm{GHz}$ desktop PC. Thus in this research efforts were made to optimize the calculations so as to reduce the computer time.

The updating method given in equation (4) consists of three main steps; (i) update the $\mathbf{A}_{\mathbf{b}}$ matrix and store the values in a temporary matrix for $\mathbf{A}_{\mathbf{b + 1}}$, (i) update the $\mathbf{B}_{\mathbf{b}}$ matrix and store the values in a temporary matrix for $\mathbf{B}_{\mathbf{b + 1}}$, and finally (iii) multiply the two temporary matrices $\mathbf{A}_{\mathbf{b + 1}}$ and $\mathbf{B}_{\mathbf{b}+\mathbf{1}}$. The first step in reducing the computer time is to write the updated resolution matrix explicitly in a single equation as follows.

$$
\mathbf{R}_{\mathbf{b}+1}=\mathbf{R}_{\mathbf{b}}+\Delta \mathbf{R}_{\mathbf{b}}, \text { where } \Delta \mathbf{R}_{\mathbf{b}}=-\frac{\mathbf{z z}^{\mathbf{T}}}{1+\mu} \mathbf{A}_{\mathbf{b}}+\mathbf{B}_{\mathbf{b}} \mathbf{g} \mathbf{g}^{\mathbf{T}}-\frac{\mathbf{z z}^{\mathbf{T}}}{1+\mu} \mathbf{g ~ g}^{\mathbf{T}}
$$

Note this scheme calculates the change in the resolution matrix $\left(\Delta \mathbf{R}_{\mathbf{b}}\right)$, rather than directly recalculate the updated resolution matrix $\mathbf{R}_{\mathbf{b + 1}}$ itself as in equation (4). The change in the resolution values (in $\Delta \mathbf{R}_{\mathbf{b}}$ ) can be several orders of magnitude smaller than resolution value itself (in $\mathbf{R}_{\mathbf{b + 1}}$ ). Thus equation (6) is less sensitive to round-off errors compared to the direct use of equation (4). The main diagonal elements of the updated resolution matrix $\mathbf{R}_{\mathbf{b}+\mathbf{1}}$ are calculated one by one, thus avoiding the use the temporary matrices $\mathbf{A}_{\mathbf{b + 1}}$ and $\mathbf{B}_{\mathbf{b + 1}}$. This not only reduces the computer memory required, but also the computer time. This new scheme avoids the need to store (and later retrieve) the elements of the temporary matrices. The floating point computational units in modern CPUs are so efficient that the time taken to transfer data values between the CPU registers and the slower main memory can be greater than the time required for the floating point multiplications and additions. 
The bulk of the numerical calculations involve matrix-vector multiplications of the form $\mathbf{z}=\mathbf{B}_{\mathbf{b}} \mathbf{g}$ and $\mathbf{y}=\mathbf{A}_{\mathbf{b}} \mathbf{z}$ (for the first term on the left hand side of the $\Delta \mathbf{R}_{\mathbf{b}}$ equation). In the computer program implementation, the values of the $\mathbf{z}$ vector are initially calculated. The computer code was optimized so that most of the time-consuming calculations can be carried out 'in situ' within the CPU floating point registers. The traffic between the CPU and main memory is thus reduced to a minimum. The time critical parts of code were written in assembly language so that the use of the available floating point CPU registers can be directly optimized (Leiterman, 2005). Each SIMD (single-instruction-multipledata) register within the CPU can store two double precision values (Gerber, 2002). The SIMD instructions that can carry out two double-precision operations with a single instruction are used. This allows the calculations of the elements of the $\mathbf{z}$ vectors for two new test configurations (each with its own $\mathbf{g}$ Jacobian vector) to be carried out simultaneously. Thus it is only necessary to move the elements of the $\mathbf{B}_{\mathbf{b}}$ matrix from the computer memory to the CPU register once for two new test configurations. The Intel Penryn CPU has four cores that can calculate the $\mathbf{z}$ vectors for four pairs of the test configurations in parallel (Chandra et al., 2001). Thus in total the calculation for eight test configurations can be made with a single transfer of the elements of the $\mathbf{B}$ matrix from the computer memory to the CPU. A similar computer code optimization is made to reduce the calculation time for the $\mathbf{y}$ (i.e. the $\mathbf{A}_{\mathbf{b}} \mathbf{z}$ product) vector. The computer time required for the remaining calculations in equation (6) that involve vector-vector multiplications is much less than that required by the matrixvector multiplications. On a $2.4 \mathrm{GHz}$ Intel Penryn Quad-Core system, the computational time required for a survey system with 30 electrodes was reduced to less than 5 minutes ( 257 seconds). This makes it practical to use the 'Compare R' method for larger survey problems.

After calculating the ranking function in equation (5) for all the test configurations, we follow the procedure used by Wilkinson et al. (2006a). Briefly, the test configuration with the highest ranking function value (denoted by the sensitivity vector $\mathbf{g}_{1}$ ) is added to the base set at each iteration. The next 
highest ranked configuration, represented by $\mathbf{g}_{2}$, is only added if it had a sufficient degree of orthogonality to the first configuration added. This is done by calculating $\left|\mathbf{g}_{\mathbf{1}} \cdot \mathbf{g}_{\mathbf{2}}\right| /\left(\left|\mathbf{g}_{1}\right|\left|\mathbf{g}_{2}\right|\right)$ and checking that it is less than a specified limit (0.97). This is repeated until a sufficient number of configurations are added to the base data set. Each new configuration is tested against those that had been previously added during that iteration only. For each iteration, the number of new configurations added is set at $0.09 n_{b}$, where $n_{b}$ is the number in the base set. After adding the new configurations, the model resolution of the new augmented base set is recalculated using equation (2). In the following discussion, this method will be referred to as the CR method.

One minor modification was made in the addition of new arrays. A check was included to ensure that the distribution of the data points in the pseudosection is symmetrical, and consequently the corresponding model resolution section pattern would also be symmetrical. As an example, a survey with 30 electrodes will have electrode positions numbered $1,2,3,4,5, \ldots 29,30$. If an array configuration (C1-C2-P1-P2) such as 2-1-4-6 is selected, the corresponding array configuration on the other half of the survey line (which is 24-26-29-30) is also selected for the optimized data set. During some iterations, this might result in the number of arrays selected to be one more than that would have been obtained without this check.

\section{Method 2 - Modified GF (BGS)}

This method was proposed by Wilkinson et al. (2006a) as an improved version of the original method by Stummer et al. (2004). The ranking function used is as follows.

$$
F_{i}^{B G S}=\sum_{j=1}^{m} \frac{G_{i j}^{2}}{\left(G_{j}^{s u m}\right)^{2}}\left(1-\frac{R_{b}(j)}{R_{c}(j)}\right)^{1 / 2}
$$

where $G_{j}^{\text {sum }}=\frac{1}{n_{c}} \sum_{k=1}^{n_{c}}\left|G_{k j}\right|$ 
$n_{c}$ is the number of arrays in the comprehensive data set and $G_{j}^{\text {sum }}$ is the sum of the absolute sensitivities for the $j$ th model cell for all configurations in the comprehensive set. $R_{b}$ and $R_{c}$ are the model resolutions for base and comprehensive data sets. The bracketed term involving $R_{b}$ and $R_{c}$ selects configurations that improve regions of the model that are poorly resolved by the base set. This equation provides a normalization factor to ensure the ranking function has equal weight in improving the resolution of all regions regardless of the relative sensitivity. The same method, as that described earlier for the CR method is used to test the configurations, and to add the new configurations to the base set. Following Wilkinson et al. (2006a), we also use a limit of 0.95 for the upper limit of the orthogonality value for the test configurations to be added to the base set. Some effort was made to optimize the computer code for this method, mainly in utilising the parallel processing capabilities of the computer system. For the test problem with 30 electrodes, this method took less than a minute (48 seconds) for 40 iterations. In the following discussion, this method will be labelled as the BGS method.

\section{Method 3 - Combined Modified GF and Compare R (BGS-CR)}

The CR method produced array configurations with the highest model resolution, while the BGS method is much faster but produced slightly lower model resolution values (Wilkinson et al., 2006a). One possible method to combine the best features of both methods is to start the search for the optimal configurations using the BGS method and end it with a few iterations of the CR method. In this way, some of the deficiencies of the BGS method can be compensated for by the CR method. The CR method is normally used for the final $20 \%$ of the iterations in this proposed combined method. As an example, if the search process takes 40 iterations, the BGS method is used for the initial 32 iterations while the CR method is used for the final 8 iterations. For the test problem with 30 electrodes, this combined method took 87 seconds (compared to 48 and 257 seconds respectively for the BGS and CR methods). 


\section{Method 4 - Original GF (ETH)}

The 'Original GF' method by Stummer et al. (2004) used the following ranking function.

$$
F_{i}^{E T H}=\sum_{j=1}^{m} \frac{\left|G_{i j}\right|}{G_{j}^{\text {sum }}}\left(1-\frac{R_{b}(j)}{R_{c}(j)}\right)
$$

In the original implementation by Stummer et al. (2004), the orthogonality check is performed against the entire base data set. Wilkinson et al. (2006a) found this criterion to be too restrictive as it discards many more add-on configurations than the less severe criteria used in the CR and BGS methods described earlier. Another disadvantage is that the calculation time is significantly longer compared to the BGS method as the orthogonality check is made against the entire base set in addition to the configurations added during that iteration. Wilkinson et al. (2006a) found that the 'Original GF' method took nearly twice as long as the 'Modified GF' (BGS) method. In an attempt to improve on this method, we use the same formula for the ranking function, but with the more limited orthogonality check used by the CR and BGS methods. We call this modification of the 'Original GF' method the 'ETH' method. The time taken by this method is almost the same as that taken by the BGS method. The difference in the model resolution values obtained by the 'Original GF' method and its modified version (ETH) is less than $3 \%$ for all iterations in several tests that we have carried out.

It is interesting to compare the ranking functions used by the BGS and ETH methods in equations (7) and (8). The BGS method uses the square of the ratio of the sensitivity value of the configuration tested to the normalized total sensitivity value for the same model cell (in the first term to the left of the summation sign) multiplied by the square root of the $\left(1-\frac{R_{b}(j)}{R_{c}(j)}\right)$ factor. In comparison, the ETH method used the first power of the ratio of the sensitivity values multiplied by the first power of the $\left(1-\frac{R_{b}(j)}{R_{c}(j)}\right)$ factor. On the basis of numerical experiments, Wilkinson et al. (2006a) found that the 
BGS method tends to select configurations that simultaneously improve the resolution whilst maintaining the orthogonality of the optimized arrays. A detailed analysis of the differences between the two methods is given in Wilkinson et al. (2006a).

\section{RESULTS}

\section{Relative resolution tests}

The first test uses a 2.5D model similar to that used by Wilkinson et al. (2006a). Briefly, the survey line has 30 electrodes with a 1 meter spacing. The subsurface model has 16 layers starting with a thickness of 0.3 meter, and the thickness of each subsequent layer was increased by $10 \%$. This results in a model with 464 model cells. All arrays with the Gamma configurations, and geometric factors greater than that for a dipole-dipole arrays with ' $a$ ' spacing of 1 meter and ' $n$ ' level of 6 were discarded. This left a comprehensive data set with 51283 unique configurations. This is marginally less than $n_{c}=51373$ used by Wilkinson et al. (2006a) who used a geometric factor of $1100 \mathrm{~m}$ (for a spacing of 1 meter) as the cutoff value, which is slightly more than the geometric factor of $1056 \mathrm{~m}$ for a dipole-dipole array with $a=1$ and $n=6$. We also use the same damping factor value of $\lambda=2.5 \times 10^{-6}$ as that used by Wilkinson et al. (2006a).

Forty iterations of each method are implemented giving a total of 4618 configurations. We show the output of the different methods in terms of the relative model resolution, $R_{r}=\frac{R_{b}}{R_{c}}$. This is the model resolution of the optimized data set divided by the resolution of the comprehensive data set. The value of $R_{r}$ lies between 0 and 1 . Figure 2 shows a plot of the average relative model resolution, $S_{r}=\frac{1}{m} \sum_{j=1}^{m} R_{r}(j)$, for each method. The CR method has the highest resolution values after the first few iterations, while the BGS method is slightly lower. The combined BGS-CR method follows the BGS curve for the first 32 iterations, and departs from it at the $33^{\text {rd }}$ iteration onwards when it switches over to 
the CR method. The model resolution value for this combined method lies in between the BGS and CR methods. The ETH method achieves significantly lower resolution values compared to the other methods. The relative model resolutions for the different methods at the $40^{\text {th }}$ iteration are $0.956(\mathrm{CR})$, 0.942 (BGS-CR), 0.920 (BGS) and 0.834 (ETH). The computer times in seconds taken by the respective methods are 257 (CR), 87 (BGS-CR), 48 (BGS) and 47 (ETH) seconds (Table 1).

Figure 2 here.

Figure 3 shows the relative model resolution sections for the initial base data set (which is the same for all the methods) and for the $16^{\text {th }}, 32^{\text {nd }}$ and $40^{\text {th }}$ iterations of the different methods. Note the initial relative resolution sections (that use the initial base data set consisting of the 147 dipole-dipole array configurations) show high relative resolution values near the surface that rapidly decreases with depth. Only the top 3.4 meters have relative resolution values of above 0.50 . For the CR method, the relative resolution values in the lower section (first column in Figure 3) rapidly increases with each iteration, and by the $40^{\text {th }}$ iteration the relative resolution values rises to above 0.95 for almost the entire section. For the BGS method, the relative resolution values also increase rapidly with each iteration, but the distribution of the high values (above 0.80) is less uniform compared to the CR method. There are patches of low values at the top left and top right regions (second column in Figure 3). These 'blind spots' are probably caused by the different ranking functions used by the BGS and CR methods. The criteria used to assess the effectiveness of the array optimization method is the model resolution. It is not surprising that the CR method performs better as it directly calculates the change in the model resolution due to the addition of a new array, whereas the BGS method uses an (heuristic) approximation in the ranking function to gauge the effect of adding a new array.

The BGS-CR method shows identical behaviour with the BGS method until the $32^{\text {nd }}$ iteration since the BGS method is used. The effect of using the CR method for the last 8 iterations is to fill up the 'blind spots' left by the BGS method. This results in a more uniform distribution of the high relative 
resolution values of above 0.925 for the entire section (third column Figure 3). The results of the ETH method is similar to that found by Wilkinson et al. (2006a). There is a significant improvement in the relative resolution values compared to the initial dipole-dipole base data set, but its performance is below that of the BGS method.

Figure 3 here.

It should be emphasized that the sections in Figure 3 show the 'relative model resolution', i.e. the ratio of the model resolution of the optimized data sets with the comprehensive data set. While the 'relative model resolution' can reach high values of over 0.9 in the lower sections of the model section (particularly for the $40^{\text {th }}$ iteration of the CR method), the 'model resolution' values at the lower sections are very low. Figure 4 shows a plot of the 'model resolution' values achieved by the different optimization methods at the $40^{\text {th }}$ iteration.

Figure $4 \mathrm{~b}$ shows the model resolution values for the comprehensive set with 51283 arrays. The model resolution section obtained with the CR method (Figure 4c) is almost identical despite having only 4618 arrays, or about $9 \%$ of the number of arrays in the comprehensive set. There are some slight differences in the sections for the BGS and ETH methods at the upper left and right sides (Figures 4d and 4f), although the differences are not as obvious as in the relative resolution sections (Figure 3). The model resolution set for the initial dipole-dipole array set shown in Figure 4a has very low values (less than 0.05 ) below a depth of 5 meters.

Figure 4 here.

Table 1 shows the computer times required by the different optimization methods for survey lines with 20 to 60 electrodes. As the number of electrodes increase, the time taken by the BGS-CR method becomes increasingly dominated by the CR method used for the last $20 \%$ of the iterations. The practical limit for the CR method is probably about 60 electrodes with present techniques. It is for these large problems that the BGS-CR method using a small number of CR iterations might prove to be the 
most useful. It takes advantage of the higher model resolution values achieved by the CR method while requiring more modest computer times.

Table 1 here.

We have also carried out tests using different values of the damping factor and model section thickness. The damping factor value of $\lambda=2.5 \times 10^{-6}$ as used by Wilkinson et al. (2006a) was chosen so that the model resolution for the comprehensive data set has values of about 0.05 at a depth of about one-fifth the survey line length. From our experience with the inversion of field data sets, much higher values of the damping factor (typically 0.001 to 0.1 ) are usually used due to noise in the data. Figures 5 , 6 and 7 show the results obtained when a value of 0.01 is used for the damping factor. One effect of using a higher damping factor is that the relative model resolution values achieved are lower. For example, after 40 iterations with the $\mathrm{CR}$ method, the average relative resolution is 0.872 compared to a value of 0.956 obtained with the lower damping factor. This occurs because equation (2) for the model resolution involves the inverse of the $\left(\mathbf{G}^{\mathbf{T}} \mathbf{G}+\lambda \mathbf{I}\right)$ term for the Marquardt-Levenberg least-squares method. It can be shown that increasing the damping factor $\lambda$ in this term leads to a decrease in the model resolution values (Golub and van Loan 1996, Menke 1989).

However, the relative performances of the different methods are generally the same. The CR method achieves the highest relative resolution values while the BGS method is close behind with an average relative resolution of 0.808 at the $40^{\text {th }}$ iteration. The combined BGS-CR method closes the gap between the two methods in the last 8 iterations reaching a value of 0.857 . The ETH method shows a steady increase up to a value of 0.688 at the $40^{\text {th }}$ iteration but lags behind the BGS method (Figure 5). The relative resolution sections for the initial base data set, $16^{\text {th }}, 32^{\text {nd }}$ and $40^{\text {th }}$ iterations are shown in Figure 6. The CR method has relative resolution values of above 0.85 for almost the entire section at the $40^{\text {th }}$ iteration. The BGS method achieves values of over 0.700 for almost the entire section, but with 
small regions with lower values near the upper left and right of the section which are filled up by the CR method during the last 8 iterations of the combined BGS-CR method (third column in Figure 6).

Figure 5 here.

Figure 6 here.

Figure 7 shows the model resolution sections for the initial base set, the comprehensive set and the base set after 40 iterations obtained by the different optimization methods using a damping factor of 0.01. The resolution sections for the comprehensive set with the lower damping factor shown in Figure $4 \mathrm{~b}$ has values of 0.05 reaching down to about 8 meters, while in the section with higher damping factor it only reaches to 6 meters (Figure $7 b$ ).

Figure 7 here.

\section{Tests with synthetic data}

In this section, we present results from tests with synthetic data from a user defined 2D model. The results for the model with four rectangular blocks used by Wilkinson et al. (2006a) are given. We have scaled the model to a unit electrode spacing of 1 meter. The model together with the pseudosection for the dipole-dipole array is shown in Figure 8. The blocks of $100 \Omega . \mathrm{m}$ are set at different depths in a medium of $10 \Omega . m$ to test the depth of investigation as well as the resolution of the different arrays. The apparent resistivity values for different types of arrays are calculated for this model using a finitedifference based forward modeling program.

Figure 8 here.

Initially the apparent resistivity pseudosections for several conventional arrays are generated. The calculated apparent resistivity data sets are then used as the input data for a $2.5 \mathrm{D}$ least-squares smoothness-constrained optimization program to generate inversion models. The $1_{1}$-norm model constraint was used since the real structure is known to have sharp boundaries (Farquharson and 
Oldenburg, 1998). Details on the computer program and Gauss-Newton least-squares optimization method used can be found in Loke and Dahlin (2002) and Loke et al. (2003).

The first test is with the Wenner array using measurements with the electrode separation ' $a$ ' ranging from 1 to 9 meters giving a total of 135 data points. The performance of this data set is rather poor (Figure 9a). The two upper blocks are reasonably well detected, but the third deepest block is rather poorly resolved. The next array is the Wenner-Schlumberger array (Pazdirek and Blaha, 1996) with overlapping data levels using the potential dipole spacing ' $a$ ' ranging from 1.0 to 9.0 meters, and using ' $n$ ' factor values of 1 to a maximum of 9, but only measurements where the geometric factors that do not exceed that for a dipole length of 1.0 meter and ' $n$ ' factor of 6 are selected (i.e. a maximum value of $1055.6 \mathrm{~m}$ when the unit electrode spacing is 1.0 meter). This data set has a total of 383 data points. The three upper blocks are better resolved (particularly the third block), but it still fails to detect the deepest block (Figure 9b). The next test data set was for the dipole-dipole array with the dipole length ' $a$ ' fixed at 1 meter, and with the dipole separation factor ' $n$ ' varying from 1 to 6 that gives a total of 147 data points (Figure 8a). The third deepest block is much better resolved compared to the Wenner and Wenner-Schlumberger arrays (Figure 9c). However, the deepest block is not detected. In an effort to improve the depth of investigation with the dipole-dipole array, measurements with different ' $a$ ' values ranging from 1 to 9 meters are used, and ' $n$ ' values ranging from 1 to 6 (but imposing the limit that the geometric factor does not exceed $1055.6 \mathrm{~m}$ ) is next used. This data set has 395 data points. The pseudosection for this data set is shown in Figure 8b. There is some indication of the deepest block in the form of an area of slightly higher resistivity between the actual locations of the third and fourth blocks. However, the inversion model fails to separate the fourth block from the third deepest block. There are also slights shifts in the locations of the first and third upper blocks. Note that in all the model sections, the thickness of the uppermost block is slightly overestimated. This could be due to the fact 
that this block is located near the left end of the survey line, and thus there are fewer measurements that cover this block.

Figure 9 here.

Figure 10 shows the inversion models obtained using the array configurations generated by the different optimization strategies. The data sets generated after the $40^{\text {th }}$ iteration was used. As expected, the best result was obtained with the CR method (with 4618 data points) where even the deepest block is clearly resolved. The shape and location of the deepest block is accurately reproduced, although the maximum resistivity of about $25 \Omega . \mathrm{m}$ is well below the true resistivity of $100 \Omega$.m. This is not unexpected as the resolution of the resistivity method decreases exponentially with depth. The deepest block is also reasonably well detected by the BGS and BGS-CR methods.

Figure 10 here.

It could be argued that better resolution of the optimized data set with 4618 data points is due to the much greater number of data points compared to the conventional arrays. Here we show the results with a much smaller optimized data set with only 413 data points (with an average relative resolution 0.768) that is similar in size to the Wenner-Schlumberger and dipole-dipole arrays with overlapping data levels (Figures 9b and 9d). It is generated using 12 iterations of the CR method. The shape of the deepest block is much better resolved (Figure 11a) compared to the dipole-dipole array, although it is slightly too shallow compared to the model obtained with the larger optimized data set (Figure 10a). However, the number of data points used is less than $10 \%$ of the larger optimized data set. Figure $11 \mathrm{~b}$ shows the resulting inversion model obtained when a moderately larger optimized data set (824 data points) generated using 20 iterations of the CR method is used. The deepest block is much better resolved. Figure 11c shows the result obtained using the smaller optimized data set generated with the BGS-CR method (using 10 iterations of the BGS method followed by 2 iterations of the CR method). The deepest block is just barely resolved although the result is still better than that obtained using the dipole-dipole 
array with overlapping data levels (Figure 9d). The deepest block is much better resolved with the medium size optimized data set (using 16 iterations of the BGS method followed by 4 iterations of the CR method). In field surveys, the practical use of the optimized data set probably lies in generating such small or medium data sets using the CR or BGS-CR methods. The data sets generated have significantly better resolutions than conventional arrays but only involve a similar number of measurements.

Figure 11 here.

Zhou and Dahlin (2003) demonstrated that the error in resistivity field measurements varies inversely with the signal strength. Thus to simulate such noise, we add Gaussian random noise (Press et al.,1992) to the potential values (for a current of 1 Ampere) for the different array configurations. After adding the noise, the potential values are converted to apparent resistivity values. The amplitude of the potential noise is chosen so that the readings with the lowest potential (and also the largest geometric factor) have a noise level of 15 percent. The largest geometric factor is about 168 times the smallest geometric factor for the arrays generated. The average percentage apparent resistivity noise depends on the geometric factors of the array configurations in the data set used, but it generally gives a value of about 2 to 4 percent. As an example, Figure 12a shows the dipole-dipole array data set with overlapping data levels with the potential dependent noise added (Figure $8 \mathrm{~b}$ shows the same pseudosection without noise). The inversion model for this data set (Figure 12b) shows slight distortions in the upper three blocks, while the area of higher resistivity at the location of the deepest block is now much less compared with the noise free model (Figure 9d). Figures $12 \mathrm{c}$ and $12 \mathrm{~d}$ shows similar models for the large optimized data sets generated by the CR and BGS-CR methods. The deepest block is still clearly visible in both sections, although the highest resistivity value reached is lower compared to the corresponding models without noise (Figures 10a and 10c). The noise added causes a slight reduction in the resolution of the deepest block.

Figure 12 here. 


\section{Field example}

The data sets presented in this field example were acquired from an active landslide site near Malton, North Yorkshire, UK. The site is being monitored using an automated time-lapse electrical resistivity tomography (ALERT) system (Kuras et al., 2009; Ogilvy et al., 2009) to study the hydraulics of landslide processes. The ALERT instrument uses wireless telemetry (in this case GPRS) to communicate with an office based PC that runs control software and a database management system. The control software is used to schedule data acquisition, whilst the database management system stores, processes and inverts the remotely streamed ERT data. The capability that this provides for flexible and remotely configurable data acquisition is ideal for testing and improving optimized command sequences without the need for repeated manual reoccupation of the site.

The research site is located on a south facing valley side with a slope of approximately $14^{\circ}$. The bedrock geology, from the base to top of slope, comprises the Lias Group Redcar Mudstone Formation (RMF), Staithes Sandstone and Cleveland Ironstone Formation (SSF), and Whitby Mudstone Formation (WMF), which are overlain at the top of the hill by the Dogger Sandstone Formation (DF). The bedrock is relatively flat lying with a gentle dip of a few degrees to the north, and the strata are broadly conformable (British Geological Survey, 1983). Slope failure at the site is occurring in the weathered WMF, which is highly prone to landsliding. The landslide is characterized by shallow rotational failures at the top of the slope that feed into larger-scale slowly moving lobes of slumped material, which extend approximately $150 \mathrm{~m}$ down the slope. The data were collected from one of five permanently installed linear electrode arrays running from the base to the top of the hill, each comprising 32 electrodes with along-line spacings of $4.75 \mathrm{~m}$. The selected array was installed along a gully between two active lobes. Two data sets were acquired, one comprising 516 dipole-dipole measurements $(a=4.75,9.5,14.25$ and $19 \mathrm{~m}$, and $n=1$ to 8 ) and another with 516 optimized measurements (using the CR method). In both cases, the maximum allowed geometric factor was $K=32233 \mathrm{~m}$ (equivalent to that for $a=14.25 \mathrm{~m}, n=$ 
8). The inverted images are shown in Figure 13. In both cases convergence was achieved after 7 iterations with extremely good fits between the measured and inverted data, indicated by mean misfit errors of $0.35 \%$ (dipole-dipole) and $0.55 \%$ (optimized).

During testing of the optimized command sequences, we observed that systematic polarization effects were being caused by the use of electrodes to measure potential that had previously been used to pass current (Dahlin, 2000; Merriam, 2005). The optimized command sequence used in Figure 13b had been reordered so that at least 3 measurements separated instances of the same electrodes being used for current and potential measurements. Without this reordering, the mean misfit error for the same set of optimized commands was $3.37 \%$. The remaining difference between the quality of the fits to the dipoledipole and optimized data sets is probably due to random noise. Although both command sequences have the same maximum geometric factor, on average the optimization process tends to select more measurements with high geometric factors. The average geometric factor for the optimized set is $5666 \mathrm{~m}$, compared to $4450 \mathrm{~m}$ for the dipole-dipole set.

Both the optimized and dipole-dipole images in Figure 13 exhibit resistivity variations consistent with the expected stratigraphic sequence. The sharp boundary between high and low resistivities near the surface in the Staithes is interpreted as the water table, which is supported by the presence of a spring line downslope from the origin at $x=-15 \mathrm{~m}$. Within the Whitby, the higher surface resistivities in the vicinity of the rotational failure are most likely due to increased localized fracturing. The positions of the water table and the Whitby / Staithes boundary have been inferred from the resistivity images, whilst the Staithes / Redcar boundary has been positioned to match the log of the auger hole at $x=-6 \mathrm{~m}$. From synthetic studies (Wilkinson et al., 2006a; 2006b), we would expect the resolution of the optimized image to be better than the dipole-dipole image at depth and towards the edges of the model. This appears to be supported by the field results, where the resistivity contours (especially the $23 \Omega . m$ contour) follow the Whitby / Staithes boundary more closely towards the north in the optimized image 
than in the dipole-dipole image. Near the surface and $x=0 \mathrm{~m}$, the optimized image also fits the water table more closely than the dipole-dipole image. The optimized image has better resolution near the ends of the survey line. This is demonstrated by the higher resolution values at the upper left and right corners of the model resolution section in Figure 4c for the CR method compared to Figure 4a for the dipoledipole array. The Staithes / Redcar boundary is less distinct than the Whitby / Staithes. The reasons for this are likely to be twofold. Firstly, in this region there is a gradational interface between the Redcar and the Staithes (Powell, 1984). Secondly, the Redcar becomes relatively silty towards the top of the formation (Rawson and Wright, 1995). Therefore it will be more lithologically, and hence electrically, similar to the Staithes.

Figure 13 here.

Figure 14 shows the distribution of the data points of the dipole-dipole array and optimized data sets. The horizontal location of the data point is calculated from the average horizontal position of the four electrodes in the array configuration while the vertical position is calculated using the median depth of investigation method (Edwards, 1977; Barker, 1989). Different symbols are used for the Alpha and Beta type of array configurations. The optimized data set has more data points in the lower part of the pseudosection (particularly below a pseudodepth of about $20 \mathrm{~m}$.) that partly accounts for the improved resolution at depth. There is also a clustering of the data points near the edges of pseudosection of the optimized data set. This is probably caused by the program attempting to improve the resolution at the left and right sides of the model section.

Figure 14 here.

\section{CONCLUSION}

The use of optimized data sets can significantly improved the resolution of $2.5 \mathrm{D}$ resistivity imaging surveys compared to conventional arrays. Among the optimization methods tested, the CR method that directly calculates the change in the resolution matrix for each new array added to the 
optimized data set gave inversion models with the highest resolution. The combined BGS-CR method gave results that are almost as good but required only about one-third to one-quarter the computer time to generate the optimized data set. The techniques described in this paper have reduced the computer time required by the $\mathrm{CR}$ method by about 80 times. This makes it practical to use the CR method to generate optimized data sets for longer survey lines with up to about 60 electrodes positions. For even longer survey lines, the BGS-CR method might prove to be the most practical alternative. Tests carried out with the small optimized data sets showed that they give significantly better resolution although the number of data points is comparable to that used with conventional arrays. The small and medium sized optimized data sets are probably the most useful in practical field surveys.

The fast methods for calculating the updated model resolution matrix developed in this paper enables the use of other criteria, such as the point spread function (Miller and Routh, 2007), for finding optimized arrays for $2.5 \mathrm{D}$ surveys. This will be studied in future research. Other possible techniques for further reducing the computer time required by the $\mathrm{CR}$ method are being investigated as part of current research in optimized arrays for 3D surveys.

\section{Acknowledgements}

This paper is published with the permission of the Executive Director of the British Geological Survey (NERC). We would like to thank two anonymous reviewers for their comments that have helped to improve the paper. 


\section{Tables}

Table 1. The times in seconds for 40 iterations (and average relative resolution ratio achieved) for the different optimization methods.

\begin{tabular}{|l|l|l|l|l|}
\hline Number of & CR method & BGS method & BGS-CR method & ETH method \\
electrodes & Time (Resolution) & Time (Resolution) & Time (Resolution) & Time (Resolution) \\
\hline 20 & $20(0.992)$ & $6(0.979)$ & $9(0.991)$ & $6(0.960)$ \\
\hline 30 & $258(0.958)$ & $48(0.920)$ & $86(0.951)$ & $47(0.837)$ \\
\hline 40 & $2635(0.921)$ & $205(0.874)$ & $680(0.909)$ & $205(0.757)$ \\
\hline 50 & $17252(0.886)$ & $668(0.840)$ & $3918(0.867)$ & $667(0.683)$ \\
\hline 60 & $62276(0.858)$ & $1745(0.808)$ & $13818(0.833)$ & $1750(0.628)$ \\
\hline
\end{tabular}

Table 2. Number of data points (and average relative resolution ratio) for the optimized data sets for a survey line with 30 electrodes at the $16^{\text {th }}, 32^{\text {nd }}$ and $40^{\text {th }}$ iterations using a damping factor of $2.5 \times 10^{-6}$. All the methods start with the same initial dipole-dipole array data set with 147 data points with average relative resolution ratio of 0.257 .

\begin{tabular}{|l|l|l|l|l|}
\hline Iteration & CR method & BGS method & BGS-CR method & ETH method \\
\hline 16 & $585(0.836)$ & $584(0.709)$ & $584(0.709)$ & $584(0.657)$ \\
\hline 32 & $2318(0.929)$ & $2317(0.876)$ & $2317(0.876)$ & $2317(0.779)$ \\
\hline 40 & $4618(0.958)$ & $4618(0.920)$ & $4617(0.951)$ & $4617(0.837)$ \\
\hline
\end{tabular}


Table 3. Number of data points (and average relative resolution ratio) for the optimized data sets for a survey line with 30 electrodes at the $16^{\text {th }}, 32^{\text {nd }}$ and $40^{\text {th }}$ iterations using a damping factor of 0.01 . All the methods start with the same initial dipole-dipole array data set with 147 data points with average relative resolution ratio of 0.145 .

\begin{tabular}{|l|l|l|l|l|}
\hline Iteration & CR method & BGS method & BGS-CR method & ETH method \\
\hline 16 & $585(0.625)$ & $584(0.494)$ & $584(0.494)$ & $585(0.454)$ \\
\hline 32 & $2318(0.802)$ & $2317(0.710)$ & $2317(0.710)$ & $2317(0.584)$ \\
\hline 40 & $4617(0.872)$ & $4618(0.808)$ & $4618(0.857)$ & $4617(0.688)$ \\
\hline
\end{tabular}




\section{References}

Barker, R.D., 1979. Signal contribution sections and their use in resistivity studies. Geophysical Journal of the Royal Astronomical Society 59, 123-129.

Barker, R.D., 1989. Depth of investigation of collinear symmetrical four-electrode arrays. Geophysics 54, 1031-1037.

Bauman, P., 2005. 2-D resistivity surveying for hydrocarbons - A primer. CSEG Recorder April 2005, 25-33.

British Geological Survey, 1983. York solid and drift (Sheet 63). 1:50 000. British Geological Survey, Keyworth, Nottingham, U.K.

Carpenter, E.W. and Habberjam, G.M., 1956. A tri-potential method of resistivity prospecting. Geophysics 11, 455-469.

Chandra, R., Dagum, L., Kohr, D., Maydan, D., McDonald, J. and Menon, R., 2001. Parallel Programming in OpenMP. Academic Press, San Diego, California, 230 pp.

Dahlin, T., 1996. 2D resistivity surveying for environmental and engineering applications. First Break $14,275-284$.

Dahlin, T., 2000. Short note on electrode charge-up effects in DC resistivity data acquisition using multi-electrode arrays. Geophysical Prospecting 48, 181-187.

Dahlin, T. and Zhou, B., 2004. A numerical comparison of 2-D resistivity imaging with 10 electrode arrays. Geophysical Prospecting 52, 379-398.

Day-Lewis, F.D., Singha, K. and Binley, A.M., 2005. Applying petrophysical models to radar travel time and electrical resistivity tomograms: Resolution-dependent limitations. Journal of Geophysical Research 110, B08206, doi:10.1029/2004JB003569.

deGroot-Hedlin, C. and Constable, S., 1990. Occam's inversion to generate smooth, two-dimensional models from magnetotelluric data. Geophysics 55, 1613-1624. 
Edwards, L.S., 1977. A modified pseudosection for resistivity and induced-polarization. Geophysics 42, 1020-1036.

Ellis, R.G. and Oldenburg, D.W., 1994. Applied geophysical inversion. Geophysical Journal International 116, 5-11.

Farquharson, C.G., and D.W. Oldenburg, 1998. Nonlinear inversion using general measures of data misfit and model structure. Geophysical Journal International 134, 213-227

Gerber, R., 2002. The Software Optimization Cookbook : High-performance recipes for the Intel architecture. Intel Press, Hillsboro, Oregon, 279 pp.

Golub, G. and van Loan, C.F., 1996. Matrix computations (Third Edition). The John Hopkins University Press, Baltimore, Maryland, 694 pp.

Kuras, O., Pritchard, J., Meldrum, P.I., Chambers, J.E., Wilkinson, P.B., Ogilvy, R.D., and Wealthall G.P., 2009. Monitoring hydraulic processes with Automated time-Lapse Electrical Resistivity Tomography (ALERT). Comptes Rendus Geosciences 341, 868-885.

Leiterman, J.C., 2005. 32/64-bit 80x86 assembly language architecture. Wordware Publishing Inc., Plano, Texas, 545 pp.

Loke, M.H. and Dahlin, T., 2002. A comparison of the Gauss-Newton and quasi-Newton methods in resistivity imaging inversion. Journal of Applied Geophysics 49, 149-162.

Loke, M.H., Acworth, I. and Dahlin, T., 2003. A comparison of smooth and blocky inversion methods in 2D electrical imaging surveys. Exploration Geophysics 34, 182-187.

Menke, W., 1989. Geophysical data analysis: Discrete inverse theory (Revised Edition). Academic Press Inc., San Diego, California, 289 pp.

Merriam, J.B., 2005. Injection electrode overprinting. Journal of Environmental and Engineering Geophysics 10, 365-370.

Miller, C.R. and Routh, P.S., 2007. Resolution analysis of geophysical images: Comparison between 
point spread function and region of influence measures. Geophysical Prospecting 55, 835-852. Ogilvy, R.D., Kuras, O., Meldrum, P.I., Wilkinson, P.B., Chambers, J.E., Sen, M., Pulido-Bosch, A., Gisbert, J., Jorreto, S., Frances, I. and Tsourlos, P., 2009. Automated Monitoring of Coastal Aquifers with Electrical Resistivity Tomography. Near Surface Geophysics 7, 367-375.

Pazdirek, O. and Blaha, V., 1996. Examples of resistivity imaging using ME-100 resistivity field acquisition system. In: EAGE 58th Conference and Technical Exhibition Extended Abstracts, Amsterdam, P050.

Powell, J.H., 1984. Lithostratigraphical nomenclature of the Lias Group in the Yorkshire basin. Proceedings of the Yorkshire Geological Society 45, 51-57.

Press, W.H., Teukolsky, S.A., Vetterling, W.T. and Flannery, B.P, 1992. Numerical Recipes in C (Second Edition). Cambridge University Press, Cambridge, UK, 994 pp.

Rawson, P.F. and Wright, J.K., 1995. Jurassic of the Cleveland Basin, North Yorkshire. In: Tayler, P.D. (Ed). Field Geology of the British Jurassic. Geological Society, London, pp. 173-208.

Seaton, W.J. and Burbey, T.J., 2000, Aquifer characterization in the Blue Ridge Physiographic Province using resistivity profiling and borehole geophysics. Journal of Environmental and Engineering Geophysics 5 (3), 45-58.

Stummer, P., Maurer, H. and Green, A., 2004. Experimental design: Electrical resistivity data sets that provide optimum subsurface information. Geophysics 69, 120-129.

Wilkinson, P.B., Meldrum, P.I., Chambers, J.C., Kuras, O. and Ogilvy, R.D., 2006a. Improved strategies for the automatic selection of optimized sets of electrical resistivity tomography measurement configurations. Geophysical Journal International 167, 1119-1126.

Wilkinson, P. B., Kuras, O., Meldrum, P. I., Chambers, J. E. and Ogilvy, R. D. 2006b. Comparison of the spatial resolution of standard and optimised electrical resistivity tomography arrays. In: 
Proceedings of the 12th meeting of the EAGE Near Surface Geophysics Conference, Helsinki, Finland, P040.

Xu, B. and Noel, M., 1991. Archaeological investigations by electrical resistivity tomography: a preliminary study. Geophysical Journal International 107, 95-102.

Zhou, B. and Dahlin, T, 2003. Properties and Effects of Measurement Errors on 2D Resistivity Imaging Surveying. Near Surface Geophysics 1, 105-117. 
Alpha

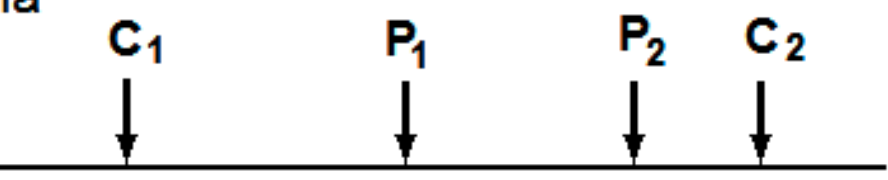

Beta

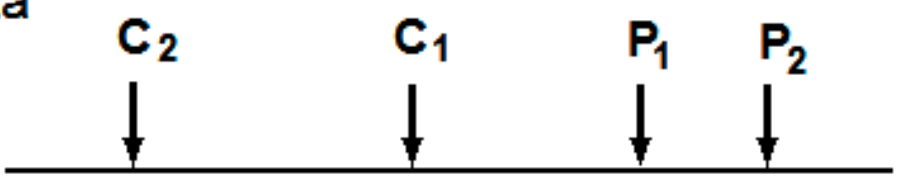

Gamma

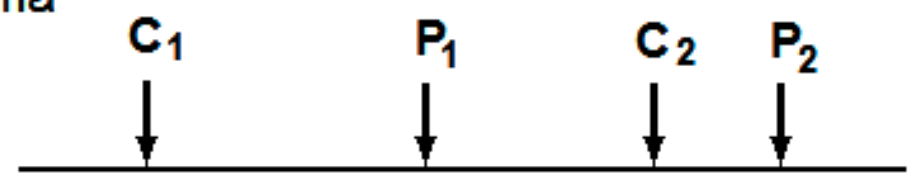

Figure 1. The three possible arrangements for an array with four electrodes. 


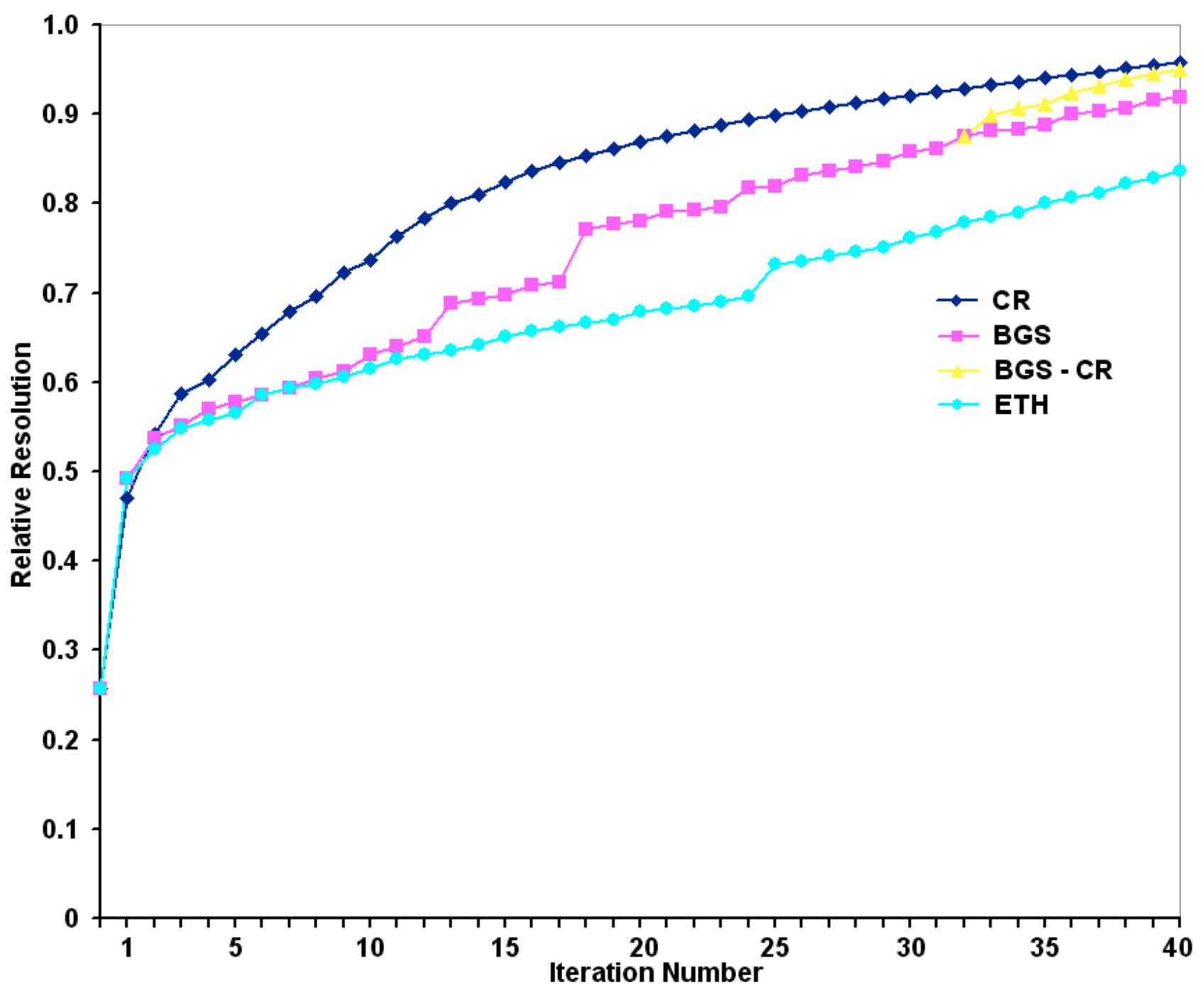

Figure 2. Change of the average relative model resolution with iteration number for the four array optimization methods. 


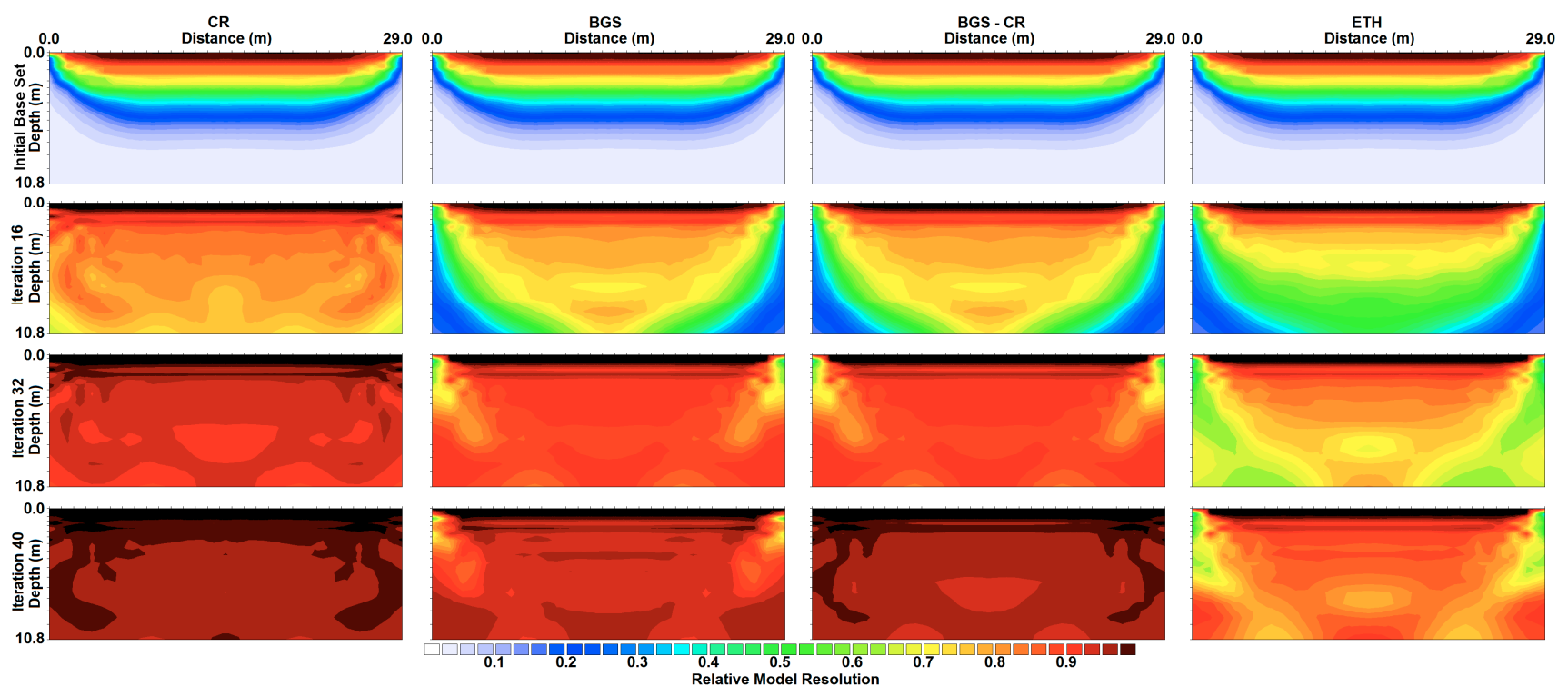

Figure 3. The top row shows the relative model resolution sections for the initial base data set with 147 dipole-dipole array configurations. The first column (starting from the top) shows the relative model resolution sections for the initial base data set, the optimized data set after the $16^{\text {th }}$ iteration, $32^{\text {nd }}$ and $40^{\text {th }}$ iterations obtained with the CR method. The second, third and fourth columns show similar results for the BGS, BGS-CR and ETH methods. The number of data points and average relative resolution values for the different methods are listed in Table 2. 


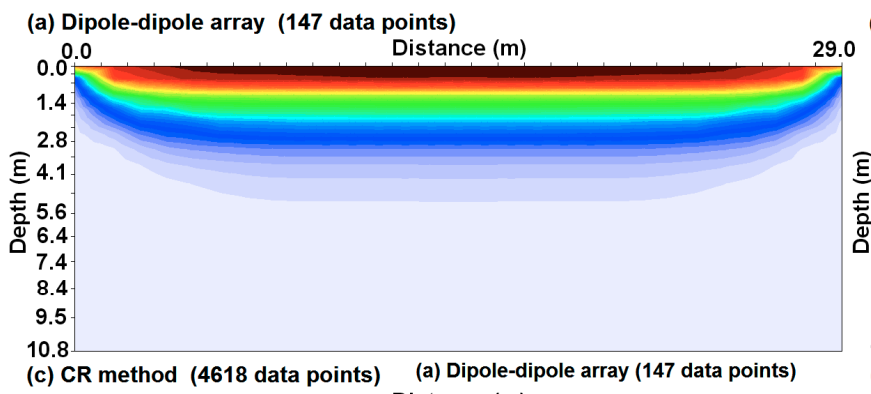

(b) Comprehensive data set (51283 data points)

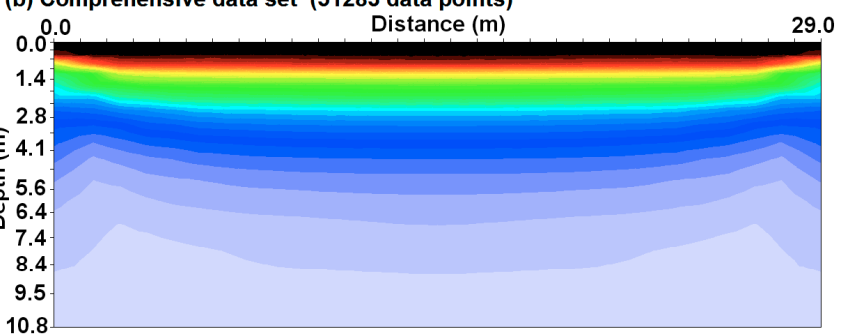

(c) CR method (4618 data points)
0.0
Distance $(\mathrm{m})$

(d) BGS method (4618 data points)

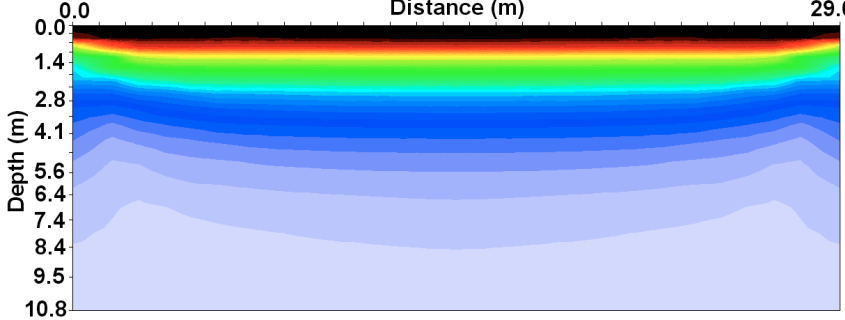

(e) BGS-CR method (4617 data points)
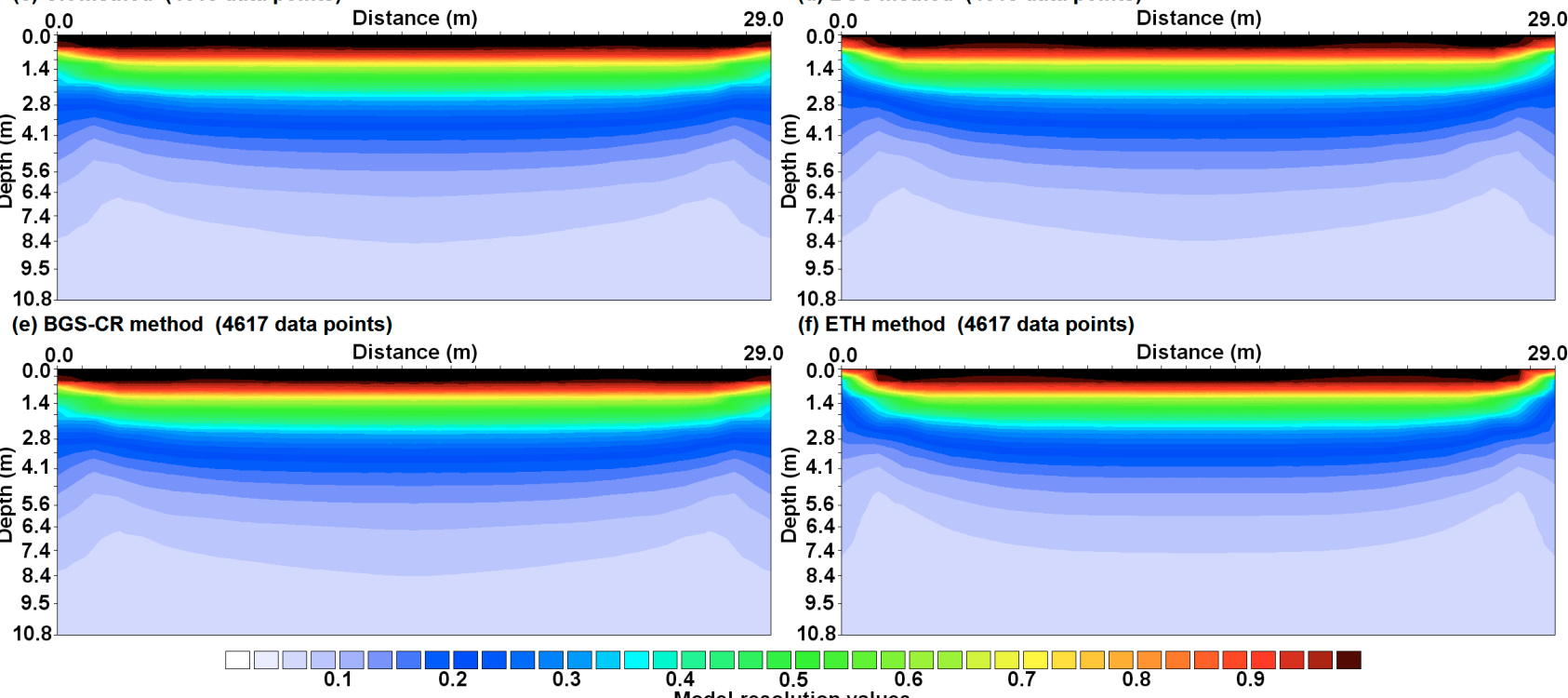

(f) ETH method (4617 data points)

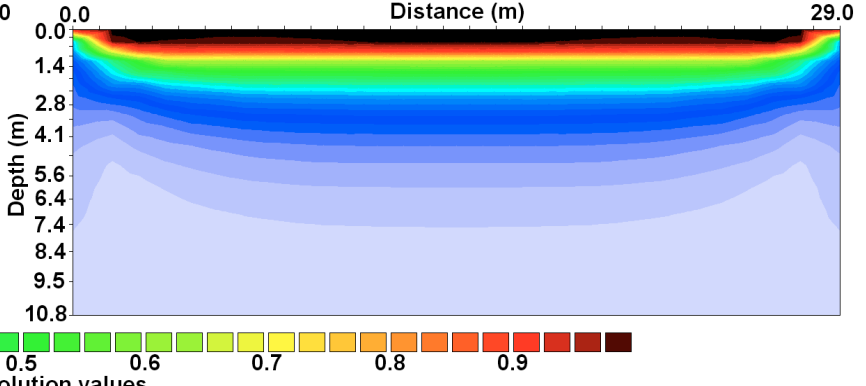

Figure 4. Model resolution values for (a) initial dipole-dipole array base data set, (b) comprehensive data set, (c) CR method, (d) BGS method, (e) combined BGS-CR method and (f) ETH method. The result for the base set generated at the $40^{\text {th }}$ iteration is shown for the different array optimization methods. 


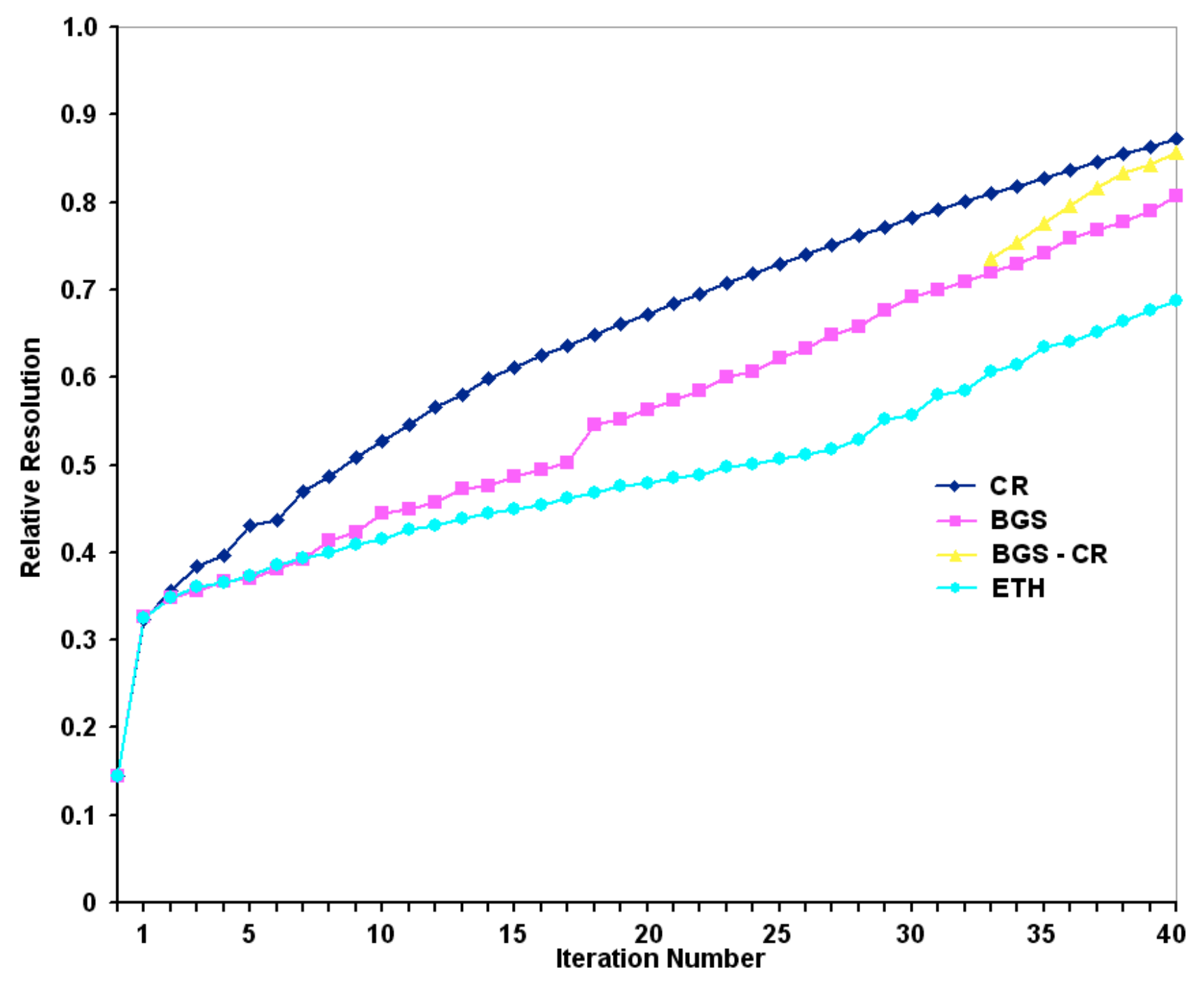

Figure 5. Change of the average relative model resolution with iteration number for the four array optimization methods when a higher damping factor of 0.01 was used. 


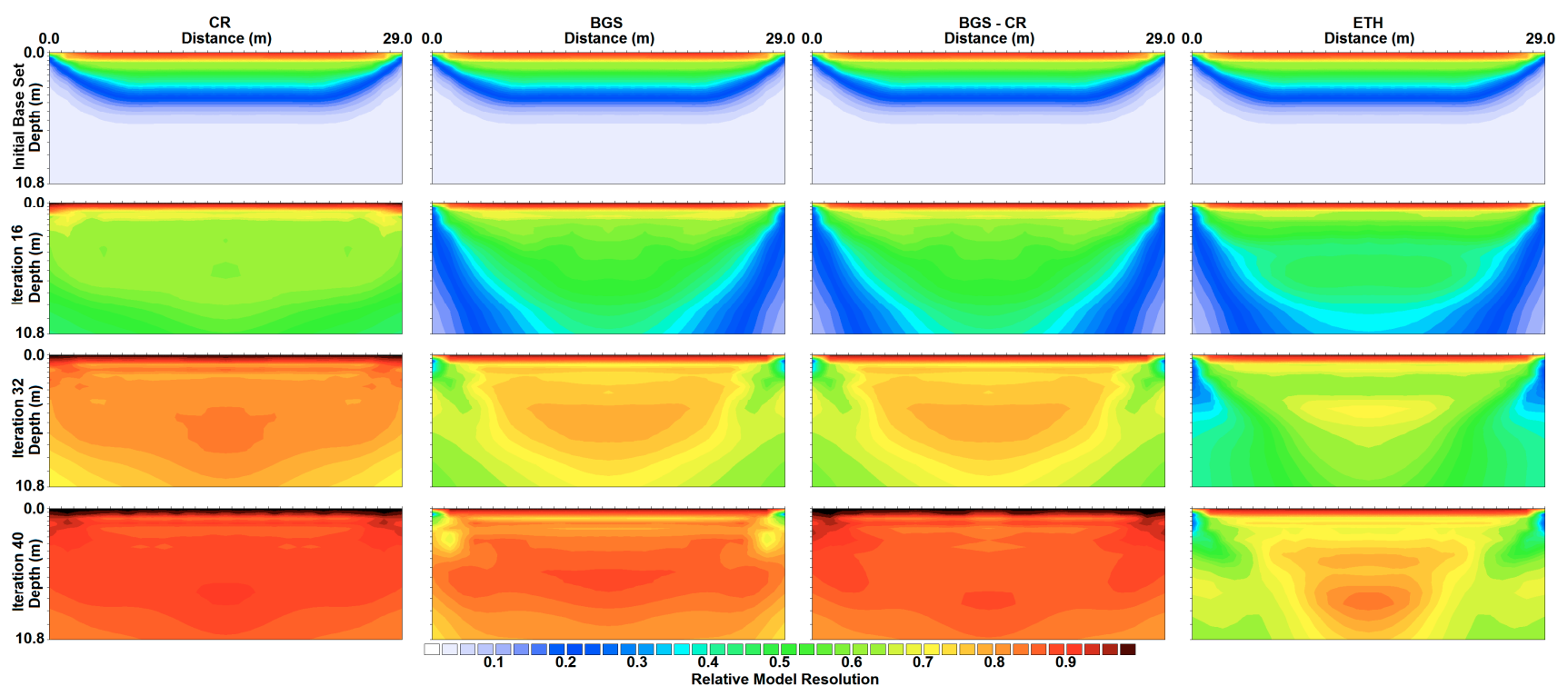

Figure 6 . The relative model resolution sections obtained when a higher damping factor of 0.01 was used. The top row shows the relative model resolution sections for the initial base data set with 147 dipole-dipole array configurations. The first column (starting second from the top) shows the relative model resolution sections for the initial base data set, the optimized data set after the $16^{\text {th }}$ iteration, $32^{\text {nd }}$ and $40^{\text {th }}$ iterations generated by the CR method. The second, third and fourth columns show similar results for the BGS, BGS-CR and ETH methods. The number of data points and average relative resolution values for the different methods are listed in Table 3. 


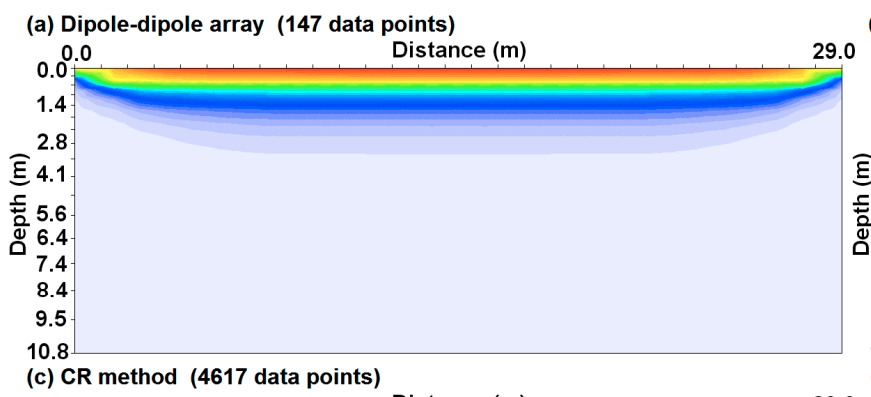

(b) Comprehensive data set (51283 data points)

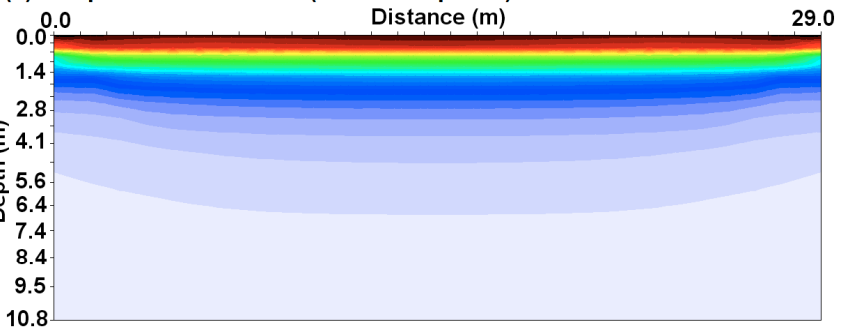

$\begin{array}{ccc}\text { (c) CR method (4617 data points) } & \\ 0.0 & \text { Distance }(\mathrm{m}) & 29.0\end{array}$

(d) BGS method (4618 data points)

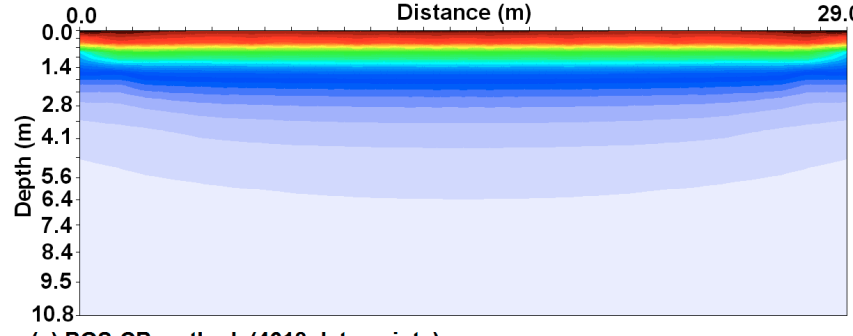

(e) BGS-CR method (4618 data points)
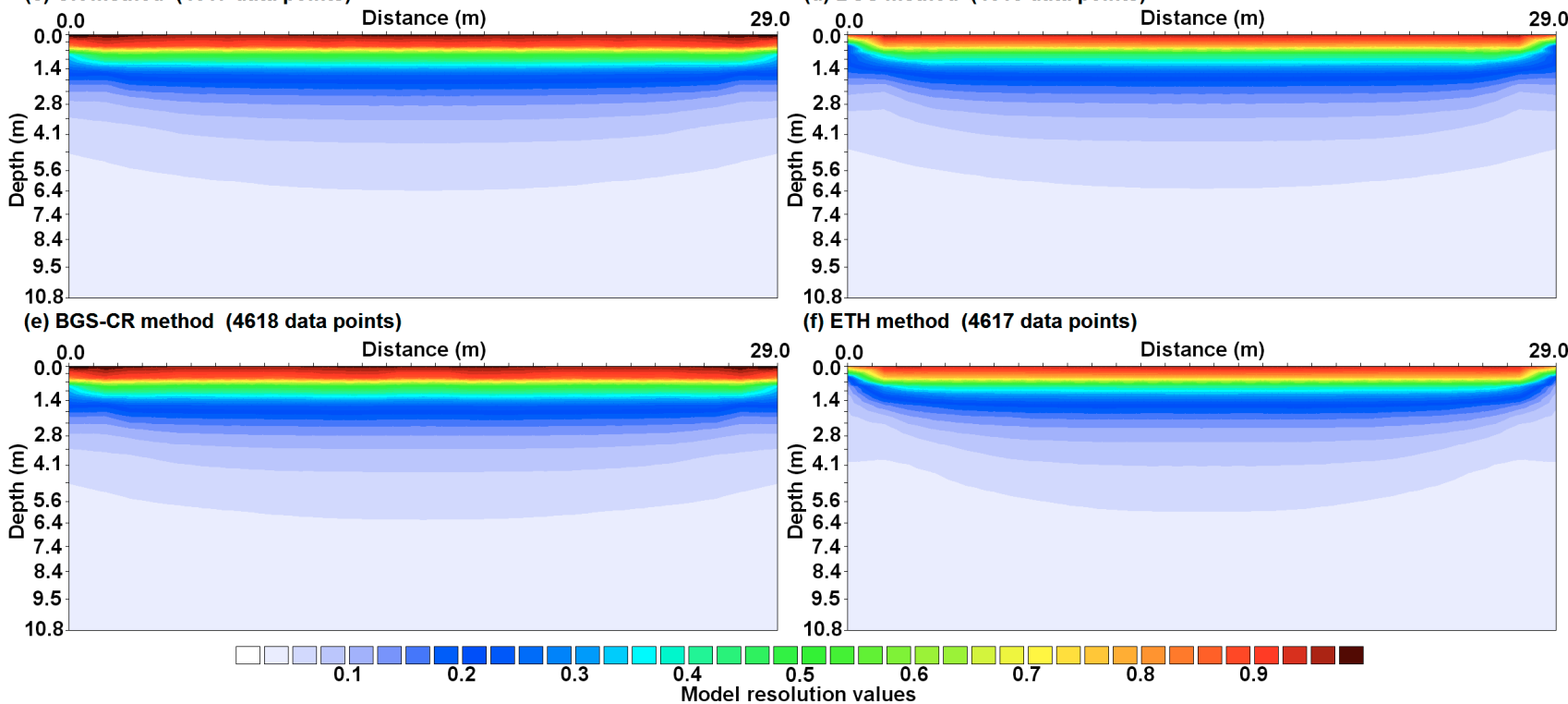

Figure 7. Model resolution values for the (a) initial dipole-dipole array base set, (b) comprehensive set,

(c) CR method, (d) BGS method, (e) combined BGS-CR method and (f) ETH method. The result for the

base set generated at the $40^{\text {th }}$ iteration is shown for the different array optimization methods using a

higher damping factor of 0.01 . 


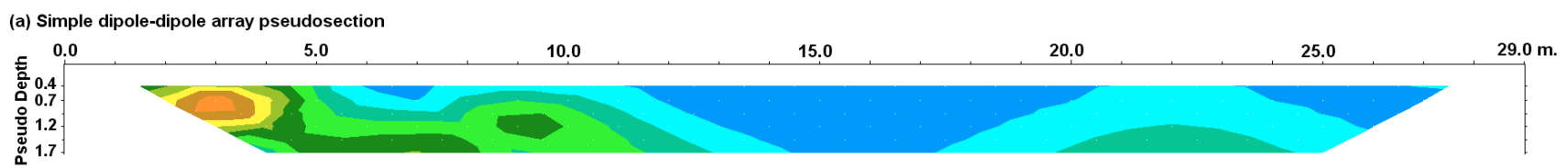

(b) Dipole-dipole array with overlapping data levels pseudosection

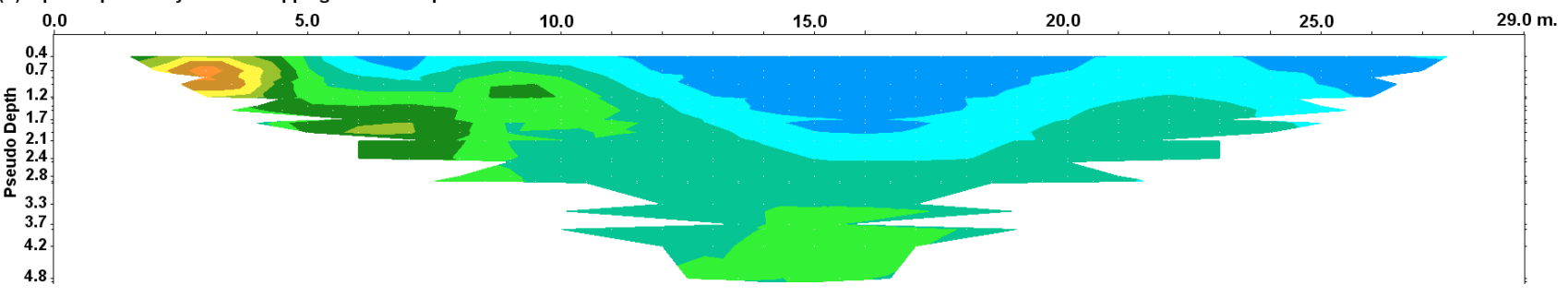

$\begin{array}{lllllllll}5.00 & 7.07 & 10.0 & 14.1 & 20.0 & \square & 28.3 & 40.0 & 56.6\end{array}$

(c) Resistivity model

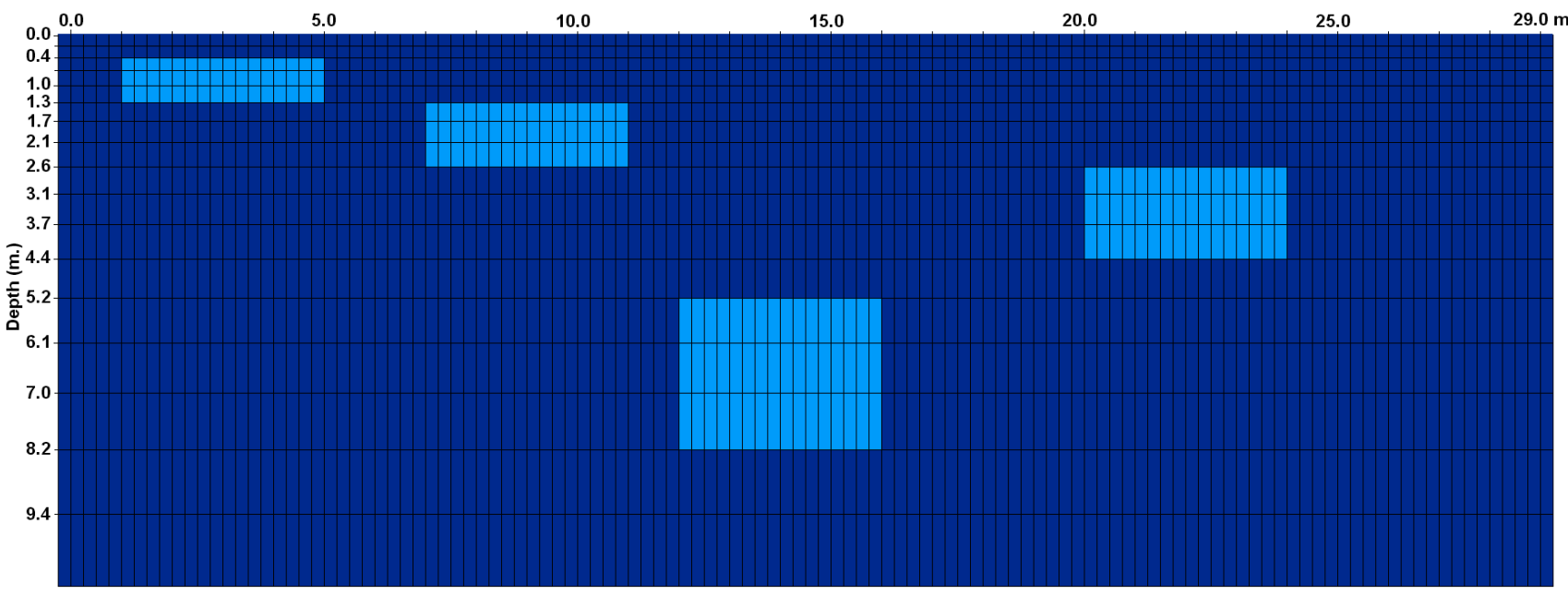

$\begin{array}{ll}\square & \square \\ 100.0\end{array}$ Resistivity in Ohm.m

Figure 8. (a) Apparent resistivity pseudosection for the simple dipole-dipole array with a dipole length ' $a$ ' of 1.0 meter with the dipole separation factor ' $n$ ' of 1 to 6. (b) Apparent resistivity pseudosection for the dipole-dipole array with overlapping data levels. (c) The synthetic model with 4 rectangular blocks of $100 \Omega . m$ embedded in a medium of $10 \Omega . m$. as used by Wilkinson et al. (2006a). 


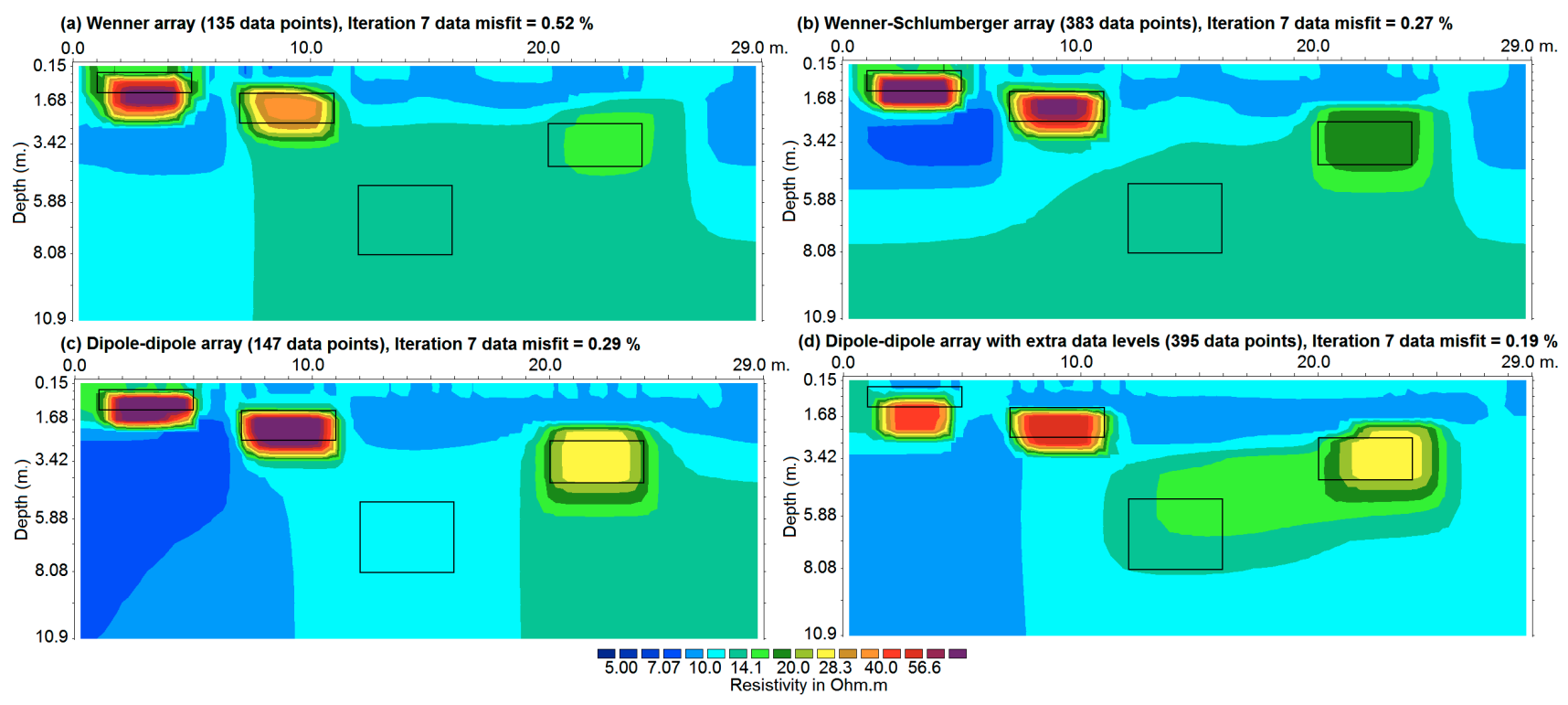

Figure 9. Inversion model obtained from the inversion of different data sets for the synthetic model with four blocks. (a) Wenner array with electrode spacings of 1.0 to 9.0 meters. (b) Wenner-Schlumberger array with overlapping data levels. (c) Simple dipole-dipole array data set with the dipole length ' $a$ ' equals to 1.0 meter, and the ' $n$ ' factor ranging from 1 to 6 . (d) Dipole-dipole array data set with overlapping data levels. The outlines of the actual rectangular blocks in the synthetic model are also shown. 


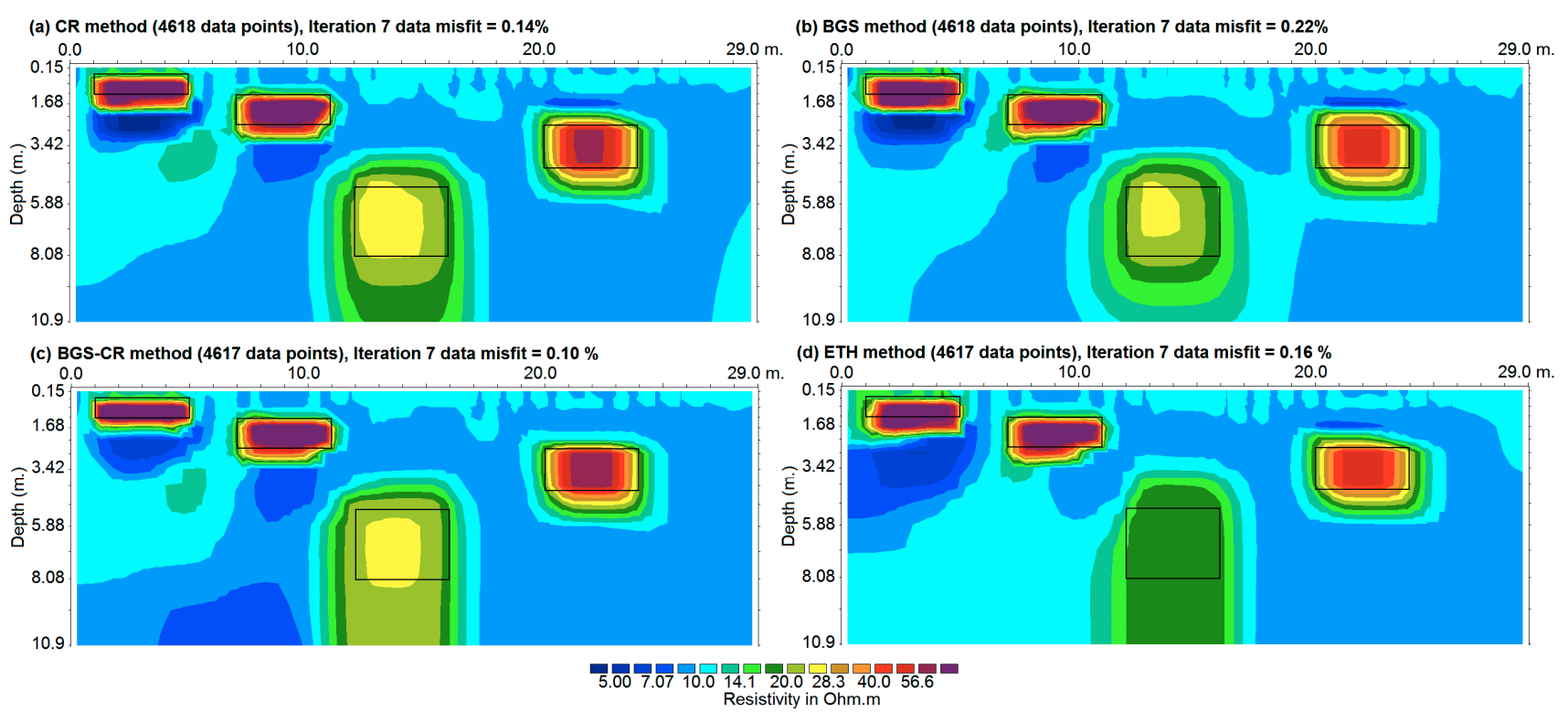

Figure 10. Inversion model obtained from the inversion of different optimized data sets (for the synthetic model with 4 blocks) generated by the (a) CR, (b) BGS, (c) BGS-CR and (d) ETH methods. 


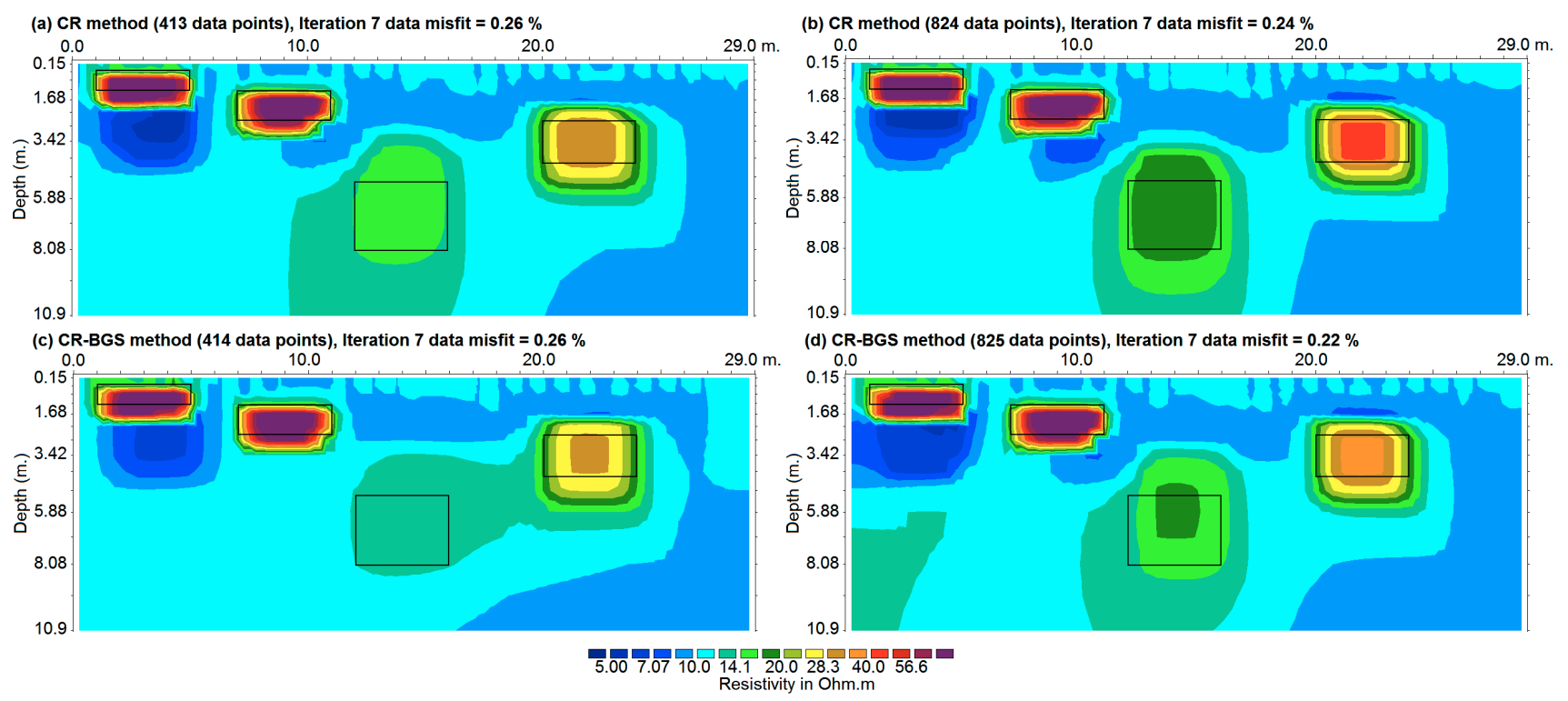

Figure 11. Inversion models obtained with the (a) small (413 data points) and (b) medium sized (824 data points) optimized data sets produced by the CR method. Inversion models for similar (c) small and (d) medium sized optimized data sets produced by the BGS-CR method. 


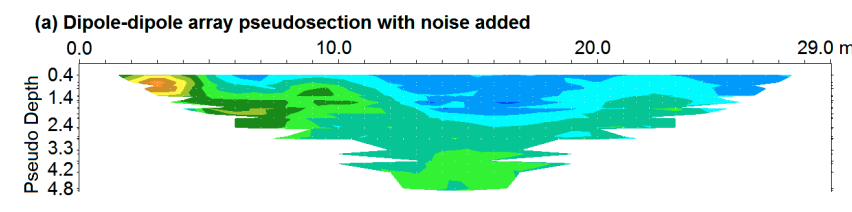

(b) Dipole-dipole array (395 data points), Iteration $\mathbf{7}$ data misfit $\mathbf{=} .54 \%$
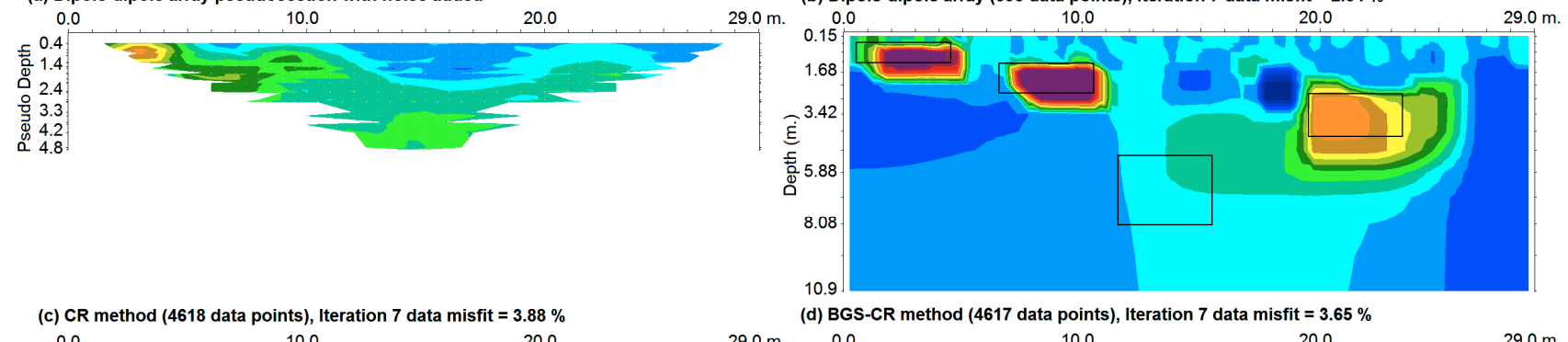
(d) BGS-CR method (4617 data points), Iteration 7 data misfit $=3.65 \%$

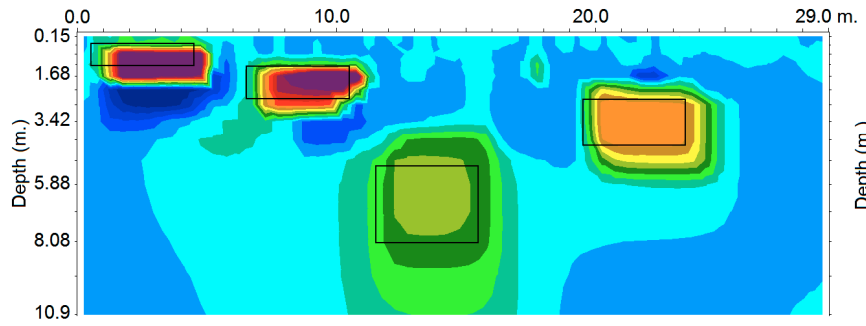

$0.0 \quad 10.0 \quad 200$ $29.0 \mathrm{~m}$.

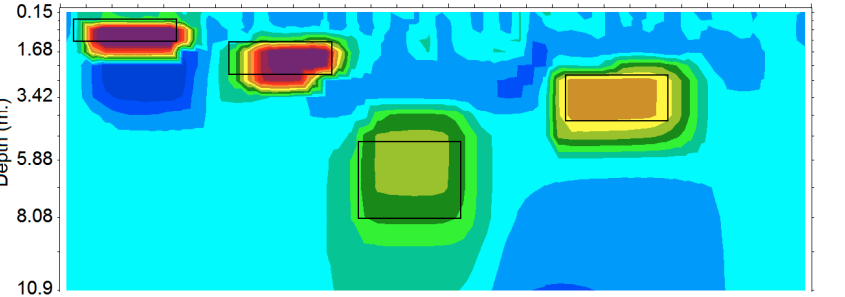

5.007 .0710 .0 14.1 20.028 .340 .056 .6

Figure 12. (a) Apparent resistivity pseudosection for the dipole-dipole array with overlapping data levels after adding the potential dependent noise. Inversion models for data sets with noise added for (b) dipole-dipole array, and optimized data sets generated by the (c) CR and (d) BGS-CR methods. 


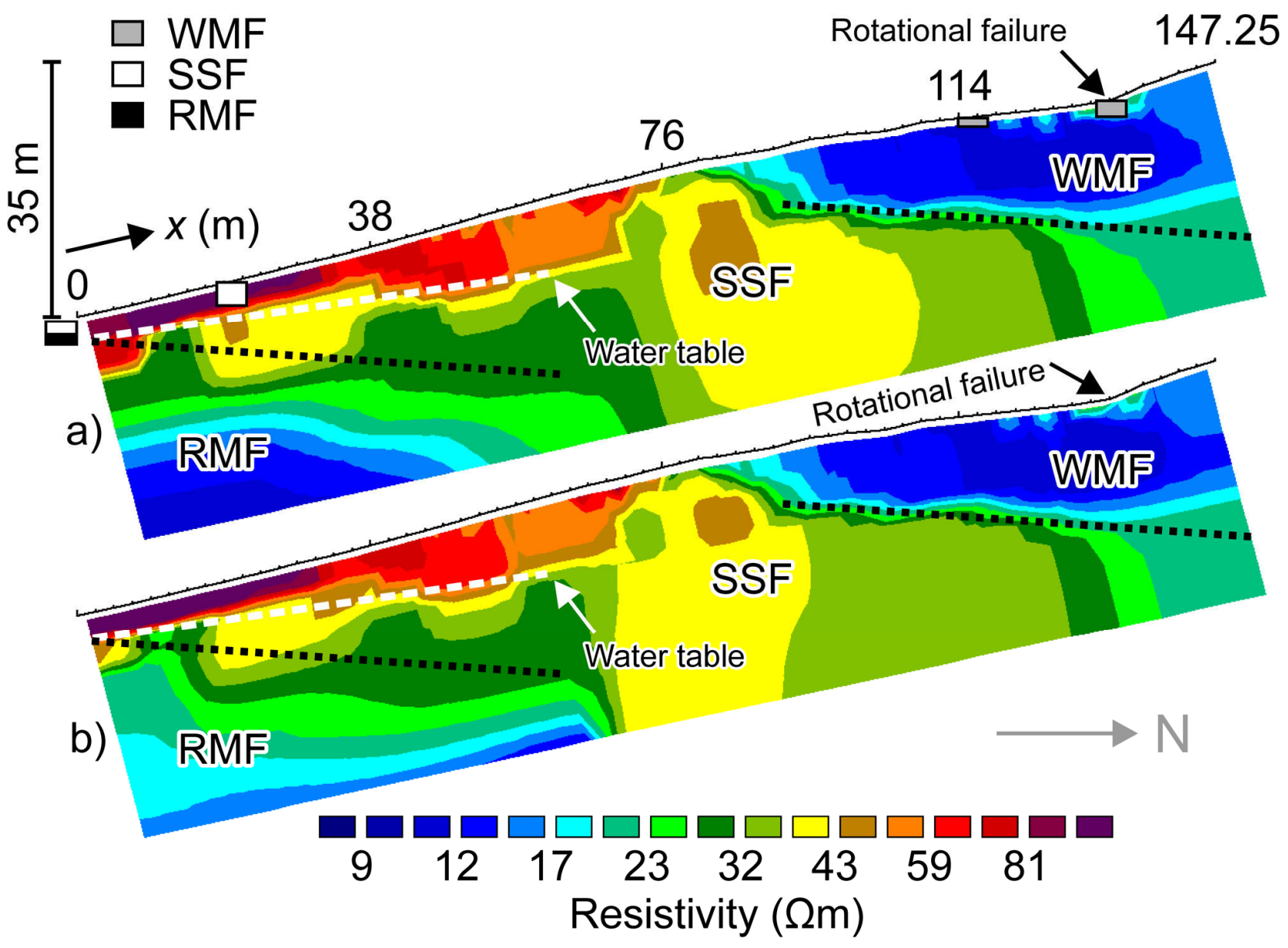

Figure 13. 2D inverted images for a) the dipole-dipole and b) the optimized data sets. The inferred boundaries between the Whitby (WMF), Staithes (SSF) and Redcar (RMF) formations are shown by dotted black lines. The inferred water table is shown by the dashed white line. Stratigraphic logs of auger holes are shown in greyscale. A rotational failure is indicated by the black arrows. North is towards the top of the slope (grey arrow). 
(a) Dipole-dipole array data set

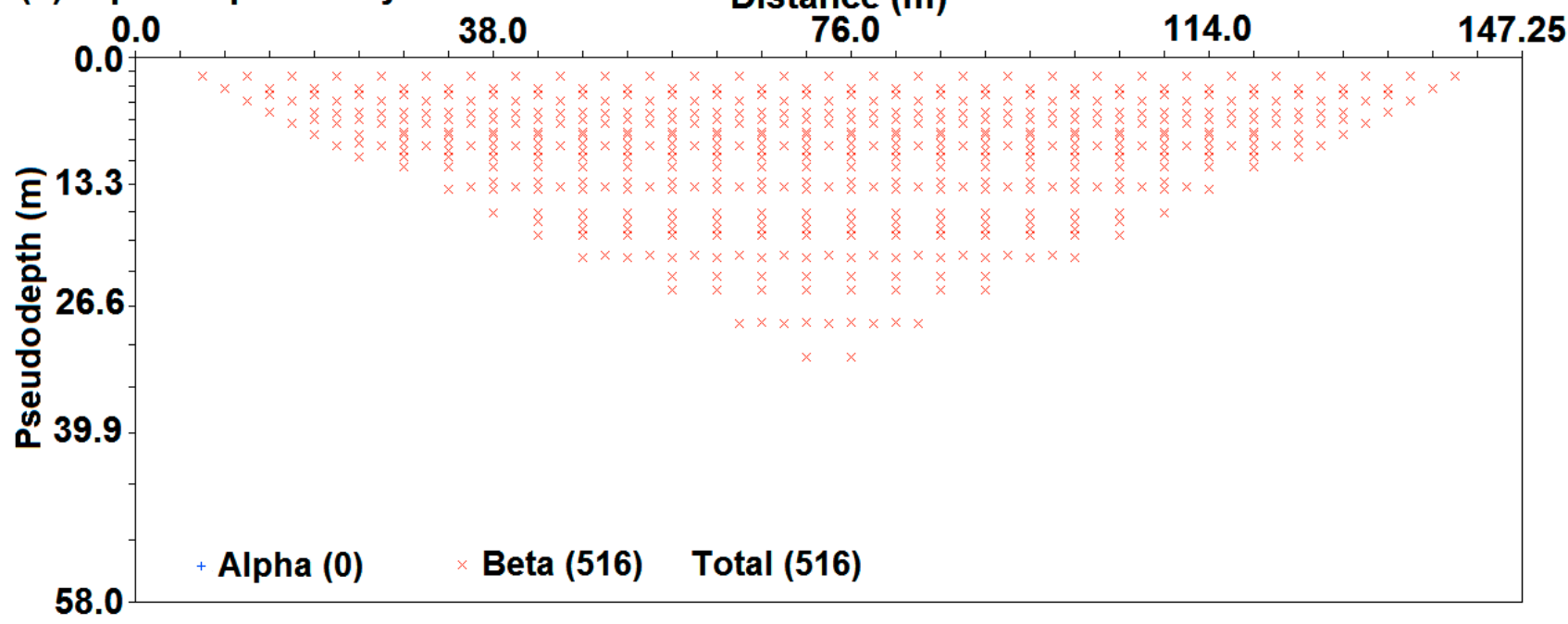

(b) Optimized arrays data set

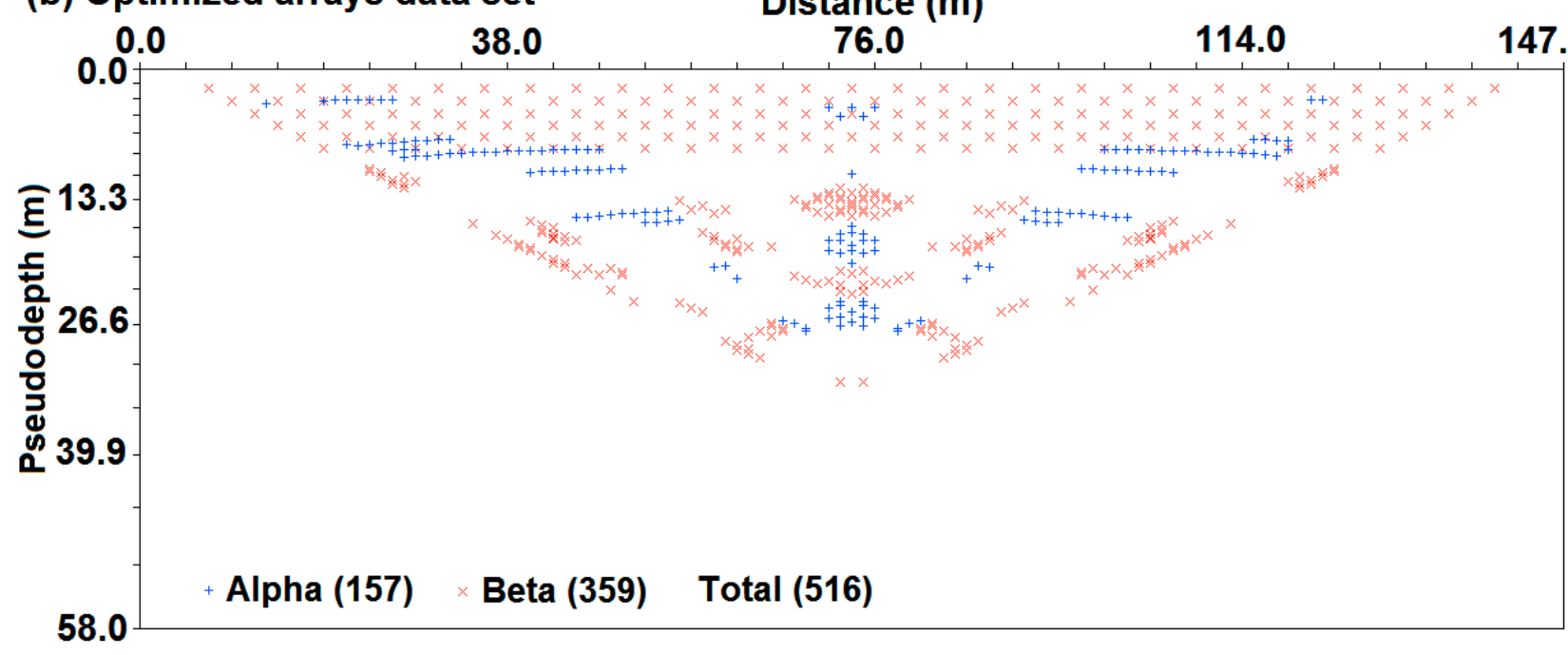

Figure 14. Distribution of data points for the dipole-dipole array and the optimized data sets used in the field survey. 